\title{
ON CERTAIN FIBRED RIBBON DISC PAIRS
}

\author{
IAIN R. AITCHISON AND DANIEL S. SILVER
}

\begin{abstract}
We prove that for any free group automorphism $\phi^{*}$ having a specified form there exists an invertible ribbon disc pair $\left(B^{4}, D^{2}\right)$ such that the closure of $B^{4}-\operatorname{nbd}\left(D^{2}\right)$ fibres over the circle with fibre a handlebody and monodromy equal to $\phi^{*}$. We apply this to obtain results about ribbon 1 - and 2 -knots.
\end{abstract}

0. Introduction and preliminaries. This paper is motivated by the question: "Does every integral polynomial $f(t)=a_{0}+a_{1} t+\cdots+a_{d} t^{d}$ with $f(1)= \pm 1$ and $a_{0} a_{d}= \pm 1$ occur as the Alexander polynomial of some fibred ribbon disc pair $\left(B^{4}, D\right)$ ?" Theorem 1 provides an affirmative answer. The proof involves handle decompositions and is very geometric. The remainder of the paper describes a consequence of this theorem: We extend to the fibred ribbon context an investigation of Burde [Bu] on classical fibred knots, and in so doing give further examples of prime fibred ribbon knots à la Quach and Weber $[\mathbf{Q u}-\mathbf{W e}]$. These have the structure expected from the (abstract) work of Casson and Gordon [Ca-Go] on fibred ribbon knots, thereby providing many examples for future study.

Throughout the paper the symbols int, cl denote interior and closure, respectively. All maps and manifolds are smooth, unless otherwise indicated. If $M$ is a manifold, then $\partial M$ denotes its boundary. If $N \subset M$ is a submanifold then $\operatorname{nhd}(N)$ denotes the tubular neighbourhood of $N$ in $M$.

An $n$-knot $(n \geq 1)$ is a submanifold $K$ of the $(n+2)$-sphere $S^{n+2}$ which is diffeomorphic to $S^{n}$. The knot is trivial if it bounds an embedded ball. We say that $K$ is fibred if $\operatorname{cl}\left(S^{n+2}-\operatorname{nhd}(K)\right)$ fibres over $S^{1}$. This means that there exists a fibre bundle $p:\left(S^{n+2}-\operatorname{int}(\operatorname{nhd}(K))\right) \rightarrow S^{1}$ such that for some identification map $f: K \times D^{2} \rightarrow \operatorname{nhd}(K),\left.p \circ f\right|_{K \times \partial D^{2}}$ is the projection onto $\partial D^{2}$. In this case, the total space $S^{n+2}-\operatorname{int}(\operatorname{nhd}(K))$ is diffeomorphic to the quotient of $V \times I$ by an equivalence $(x, 0) \sim(\phi(x), 1)$, where $V$ is an $(n+1)$-manifold and $\phi$ is a diffeomorphism. The manifold $V$ is called a fibre. The automorphism $\pi_{1}(V)$ induced by $\phi$, well defined up to conjugation in $\operatorname{Aut}\left(\pi_{1}(V)\right)$, is called the monodromy of $K$. Any $n$-knot is said to be a ribbon $n$-knot of $m$-fusions if $K$ has the form

$$
S_{0}^{n} \cup S_{1}^{n} \cup \cdots \cup S_{m}^{n} \bigcup_{i=1}^{m} f_{i}\left(\partial D^{n} \times I\right)-\operatorname{int}\left\{\bigcup_{i=1}^{m} f_{i}\left(D^{n} \times \partial I\right)\right\}
$$

where $S_{0}^{n} \cup S_{1}^{n} \cup \cdots \cup S_{m}^{n}$ is a trivial link of $m$ components (i.e. a collection of trivial $n$-knots separated by disjoint $(n+1)$-spheres) and $f_{i}: D^{n} \times I \rightarrow S^{n+2}(1 \leq i \leq m)$

Received by the editors September 13, 1986.

1980 Mathematics Subject Classification (1985 Revision). Primary 57M25, 57Q45; Secondary $57 \mathrm{~N} 13$.

Second author partially funded by the University of South Alabama Research Committee. 
are disjoint embeddings such that

$$
f_{i}\left(D^{n} \times I\right) \cap S_{j}^{n}= \begin{cases}f_{i}\left(D^{n}, 0\right) & \text { if } j=0, \\ f_{i}\left(D^{n}, 1\right) & \text { if } j=i, \\ \phi & \text { otherwise }\end{cases}
$$

By ribbon knot we mean a ribbon 1-knot of $m$ fusions for some $m$; i.e., a bandconnect-sum of an $(m+1)$-component unlink. For general information about $n$ knots, fibred $n$-knots and ribbon $n$-knots the reader may consult [Ke-We, Ya and Ma].

An $n$-disc knot $(n \geq 1)$ is a submanifold $D$ of the $(n+2)$-ball $B^{n+2}$ which is diffeomorphic to $B^{n}$. We call $\left(B^{n+2}, D\right)$ a disc pair. Taking boundaries, the $n$-disc pair gives rise to an $(n-1)$-knot $\left(\partial B^{n+2}, \partial D\right)=\left(S^{n+1}, \partial D\right)$ which we refer to as $\partial D$. We say that the pair is fibred if $\operatorname{cl}\left(B^{n+2}-\operatorname{nhd}(D)\right)$ fibres over $S^{1}$, as above: the boundary is a fibred knot. The monodromy of the fibred disc pair is defined as above. The disc pair is invertible if there exists another disc pair $\left(B^{n+2}, D_{0}\right)$ such that $\partial D=\partial D_{0}$, and the $n$-knot created by the union $D^{n} \cup D_{0}^{n} \subset B^{n+2} \cup B^{n+2}=$ $S^{n+2}$ is trivial. In this case, the $(n-1)$-knot $K=\partial D$ is said to be doubly null cobordant or doubly slice $(n>1)$. Finally, $D$ is called a ribbon disc if the radial map $B^{n+2} \rightarrow I$, restricted to $D$, is a Morse function with critical points of index 0 and 1 only, and in which case $\partial D$ is called a ribbon knot. See $[\mathbf{H i}, \mathbf{S u}, \mathbf{L e}]$ for further information about $n$-disc knots.

We wish to thank Andrew Casson, Patrick Gilmer and the referee for their suggestions. The second author wishes to thank the mathematics department at U.C. Berkeley for their hospitality during the summer of 1984 .

1. Statement of results. Let $F$ denote the free group on $x_{i}, i=1, \ldots, d$. Consider automorphisms $\phi^{*}$ of $F$ of the form

$$
\phi^{*}\left(x_{i}\right)= \begin{cases}x_{i+1} & \text { if } 1 \leq i<d, \\ w=u x_{1}^{\varepsilon} v^{-1} & \text { if } i=d(\varepsilon= \pm 1) .\end{cases}
$$

Here, $u$ and $v$ are arbitrary words (possibly empty) in $F$ that do not contain $x_{1}^{ \pm 1}$. Let $\sigma\left(\phi^{*}\right)$ denote the total exponent sum of $x_{1}, \ldots, x_{d}$ in $w$.

THEOREM 1. Let $\phi^{*}$ be an automorphism of $F$ having form $(*)$ and $\sigma\left(\phi^{*}\right)=0$ or 2. There exists an invertible fibred ribbon disc pair $\left(B^{4}, D\right)$ with fibre a genus $d$ handlebody and monodromy equal to $\phi^{*}$.

As a corollary we obtain the followin result for classical knots: (See $[\mathbf{Q u}, \mathbf{Q u}-$ We] for related results.)

THEOREM 2. Let $f(t)=a_{0}+a_{1} t+\cdots+a_{d} t^{d}$ be any polynomial with integer coefficients such that $f(1)= \pm 1$ and $a_{0} a_{d}= \pm 1$. There exists a doubly slice fibred ribbon knot $k \subset S^{3}$ with Alexander polynomial $\Delta_{k}(t)$ equal to $f(t) f\left(t^{-1}\right)$. In fact, $k$ is the boundary of a fibred ribbon disc in $B^{4}$ with Alexander polynomial $f(t)$.

REMARKS. 1. In [Fo-Mi] Fox and Milnor showed that the Alexander polynomial of any ribbon (or slice) knot $k \subset S^{3}$ has the form $f(t) f\left(t^{-1}\right)$ (where $f(1)= \pm 1$ ). If the knot is fibred, we must also have $a_{0} a_{d}= \pm 1$. Consequently, Theorem 2 realizes all possible Alexander polynomials for fibred ribbon knots. 
Previously, Terasaka $[\mathbf{T e}]$ had shown that for any integral polynomial $f(t)$ with $f(1)= \pm 1, f(t) f\left(t^{-1}\right)$ is the Alexander polynomial of some ribbon knot in $S^{3}$. This knot is the boundary of a ribbon disc in $B^{4}$ with Alexander polynomial $f(t)$.

2. Until now the only known constructible class of doubly slice fibred ribbon knots was for $f(t)$ a knot polynomial with $a_{0} a_{d}= \pm 1$. The construction in this case is as follows: Burde [Bu] has shown how to construct a fibred knot $l$ whose polynomial is the given suitable $f(t)$. It is well known that $l \#-l$ is also fibred, with Alexander polynomial $f(t) f\left(t^{-1}\right)$. Furthermore, Zeeman $[\mathbf{Z e}]$ has shown that every such knot is doubly slice. That it is a ribbon knot is also easily seen. Hence the significant advance in our result is allowing $f(t)$ to be asymmetric, in which case the knots we construct are necessarily prime if $f(t)$ is irreducible. (Asymmetry of $f(t)$ is not sufficient to guarantee the construction of a prime knot, as is clear by consideration of the case $f(t)=h(t) h(t)$.)

2. Proof of Theorem 1. Let $\Xi_{d}$ denote a genus $d$ handlebody in $S^{3}$. ( $\Xi_{d}=$ $\#_{d}^{\partial} S^{1} \times D^{2}$, the boundary connect-sum of $d$ solid tori.) The fundamental group of $\Xi_{d}$ will be identified with the free group $F$ on $x_{1}, \ldots, x_{d}$ in the usual manner. We explicitly construct a diffeomorphism $\phi$ of $\Xi_{d}$ such that the monodromy of the mapping torus is the induced automorphism $\phi^{*}$ of $F$. We then show that the mapping torus $M_{\phi} \cong \Xi_{d} \times[-1,1] /(x,-1) \sim(\phi(x), 1)$ is diffeomorphic to $\operatorname{cl}\left(B^{4}-\operatorname{nhd}(D)\right)$, where $\left(B^{4}, D\right)$ is a ribbon disc pair.

3. The diffeomorphism $\phi$. Begin with the handlebody $\Xi_{d}$ standardly embedded in $S^{3}=R^{3} \cup\{\infty\}$ as in Figure 1. The handlebody is regarded as the union of regular neighbourhoods of $d$ concentric circles $c_{1}, \ldots, c_{d}$ in the $x y$-plane (consecutively numbered with $c_{1}$ innermost) centred at the origin, and a ball $B$ with centre on the negative $x$-axis. Let $A_{i}$ denote an annular neighbourhood of $c_{i}$ contained in the $x y$-plane, $1 \leq i \leq d$, with the $A_{i}$ 's disjoint.

We describe an isotopy in $S^{3}$ of $\Xi_{d}$ which leaves a 3-ball $B^{\prime} \subset B$ pointwise fixed throughout (Figure 2), with $B^{\prime} \cap \partial B$ a 2-disc on the upper $(z>0)$ side of $B$. The desired diffeomorphism will be defined as the time 1 (i.e. final) map of this isotopy. It is helpful to regard the ball $B^{\prime}$ as a neighbourhood of a chosen 0-spine for $\Xi_{d}$. The 0 -spine has been stretched to an arc $\mu$, along the $x$-axis, which meets each circle $c_{i}$ in a single point. Henceforth the arc $\mu$ will serve as a "basepoint" for the fundamental group of $\Xi_{d}$. Let $x_{i}$ denote the homotopy class of the circle $c_{i}$, oriented as in Figure $1,1 \leq i \leq d$. Then $\pi_{1}\left(\Xi_{d}, \mu\right)$ is a free group on $x_{1}, \ldots, x_{d}$.

The isotopy will now be described by regarding $\Xi_{d}$ as the 3-ball $B$ with $d 1$ handles $h_{1}, \ldots, h_{d}$ attached and "sliding" the feet of the handles appropriately. More precisely: Each $h_{i}=\left(\operatorname{nhd}\left(c_{i}\right)-B\right)$ is attached to $B$ along 2-discs $D_{i}^{+}, D_{i}^{-}$as indicated in Figure 2. To "slide" $D_{i}^{+}\left(\right.$resp. $\left.D_{i}^{-}\right)$means to isotope $D_{i}^{+}\left(\operatorname{resp} . D_{i}^{-}\right)$ through a collar neighbourhood of $\partial\left(B \cup h_{1} \cup \cdots \cup h_{i-1} \cup h_{i+1} \cup \cdots \cup h_{d}\right)$ and then to extend the isotopy over $h_{i}$ (See Figure 3.)

We keep track of the images of the annuli $A_{j}$ during the isotopy, since these determine the framings for the attaching of 2-handles in the construction of the mapping torus $M_{\phi}$.

We must consider two cases: $\varepsilon= \pm 1, \varepsilon=-1$. For each case, we describe the isotopy in general, but illustrate the special case:

$$
d=4, \quad u=x_{3}^{2} x_{2}^{-1} x_{3}^{2} x_{4} x_{3}^{-1}, \quad v=x_{4} x_{2}^{-1} x_{4} x_{2} x_{4} .
$$




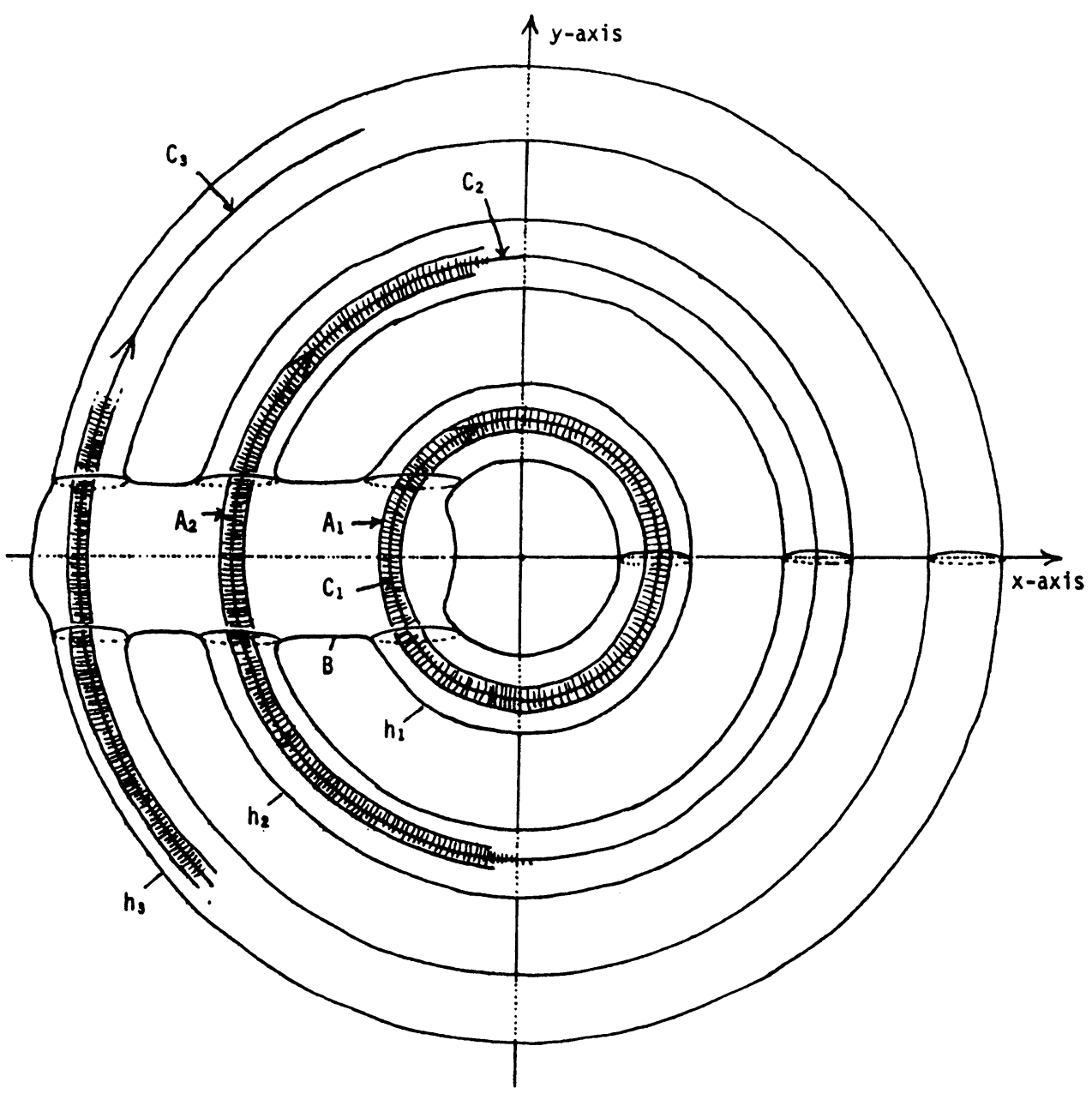

FIGURE 1

Note that in this example $\sigma\left(\phi^{*}\right)=1$.

Case I: $\varepsilon=+1$.

Step 1. Slide $D_{i}^{+}$(resp. $D_{i}^{-}$) to the present position of $D_{i+1}^{+}\left(\operatorname{resp} . D_{i+1}^{-}\right)$, $1 \leq i \leq d$. Slide $D_{d}^{ \pm}$to the end of $B$, as in Figure 4 .

Step 2. Slide $D_{d}^{-}$to the original position of $D_{1}^{-}$, staying underneath $B$ (i.e. $z<0$ ). This has the effect of stretching $c_{d}$ under $c_{1}, \ldots, c_{d-1}$ (Figure 5). For convenience we have drawn $\Xi_{d}$ with only part of the handles $h_{1}, \ldots, h_{d-1}$ indicated; we will not slide these handles henceforth.

Step 3. Slide $D_{d}^{+}$on the lower side of $B \cup h_{1} \cup \cdots \cup h_{d-1}$ according to the word $u$, read left to right, as in Figure 6 . Note that the circle $c_{d}$ is wrapped around $\Xi_{d}$. Each strand of $c_{d}$ is placed to the right of those strands of $c_{d}$ that have already been laid around a given handle. Also, each strand is placed underneath all strands previously laid. Slide $D_{d}^{+}$to the position it occupied at the beginning of this step. 


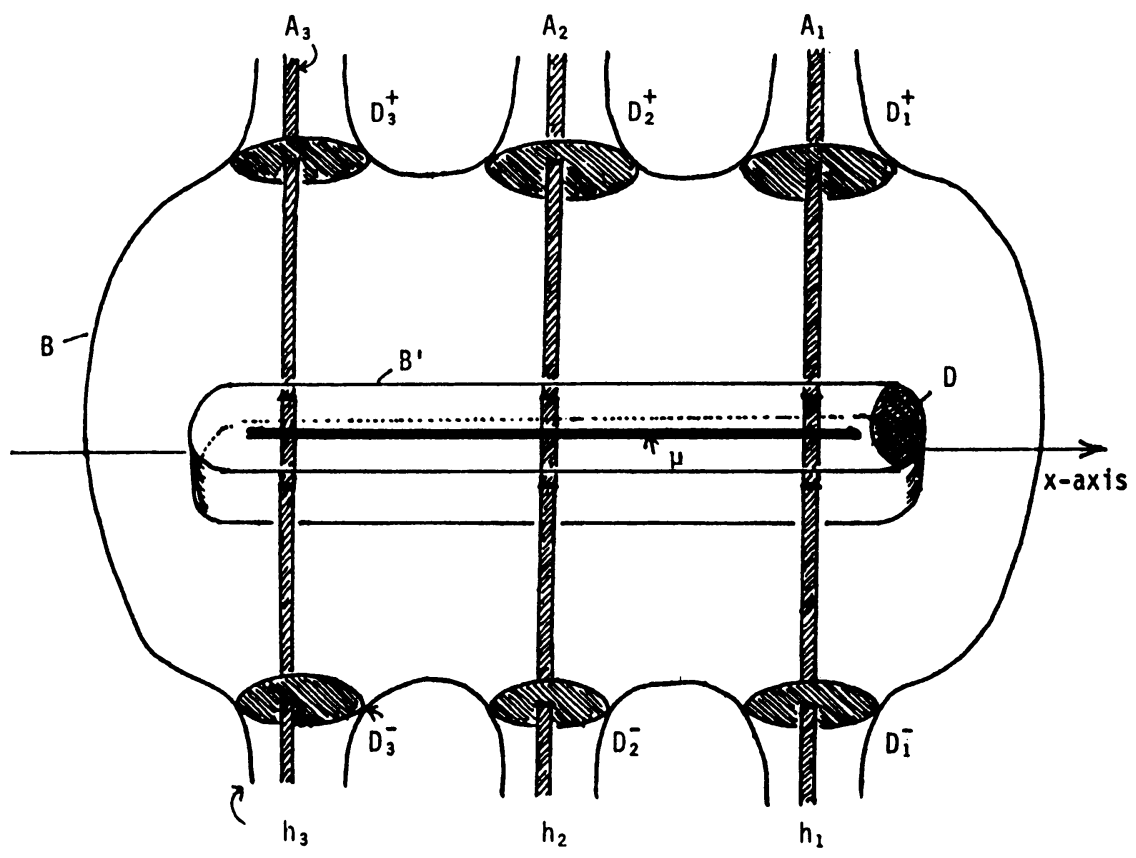

FIGURE 2

Step 4. Slide $D_{d}^{-}$according to the word $v$, read left to right, in the manner of Step 3. Slide $D_{d}^{-}$to the position that is occupied at the beginning of this step (Figure 7).

Step 5. Slide $D_{d}^{+}$underneath to the original position of $D_{1}^{+}$(Figure 8). We require that the $A_{i}$ are untwisted as they pass around the 1-handles $h_{j}$.

Steps 1-5 describe an isotopy in $S^{3}$ of the handlebody $\Xi_{d}$. Let $\phi$ denote the time 1 map of this isotopy. Note that $\phi$ is the restriction of a diffeomorphism $\Phi: S^{3} \rightarrow S^{3}$ which is isotopic to the identity.

Case II. $\varepsilon=-1$.

Step $1^{\prime}$. As in Case I, followed by a $180^{\circ}$ twist of $h_{d}$ (Figure 9). The direction indicated is crucial.

Step $2^{\prime}$. Slide $D_{d}^{-}$to the original position of $D_{1}^{+}$, staying underneath $B$.

Step $3^{\prime}$. Slide $D_{d}^{+}$on the lower side of $B \cup h_{1} \cup \cdots \cup h_{d-1}$ according to the word $u$ and return.

Step $4^{\prime}$. Slide $D_{d}^{-}$according to the word $v$ and return, staying on the underside of $\Xi_{d}$.

Step $5^{\prime}$. Slide $D_{d}^{+}$underneath to the initial position of $D_{1}^{-}$.

4. The mapping torus $M_{\phi}$. We use the technique introduced in Akbulut and Kirby $[\mathbf{A k}-\mathbf{K i}]$. Further details and applications are included in $[\mathbf{M o}, \mathbf{A i}-\mathbf{R u}$ and $\mathbf{A i}$ ], and so we are brief in our description. We continue to illustrate the special case above. However, the remaining arguments apply to both cases $\varepsilon=+1$ and $\varepsilon=-1$.

Step 1. Consider a model for $\Xi_{d} \times[-1,1]$ by first considering a 0 -handle $B \times[-1,1]$ whose boundary 3 -sphere we view as $B \times\{-1\} \cup \partial B \times[-1,1] \cup B \times\{1\}$. Regard 

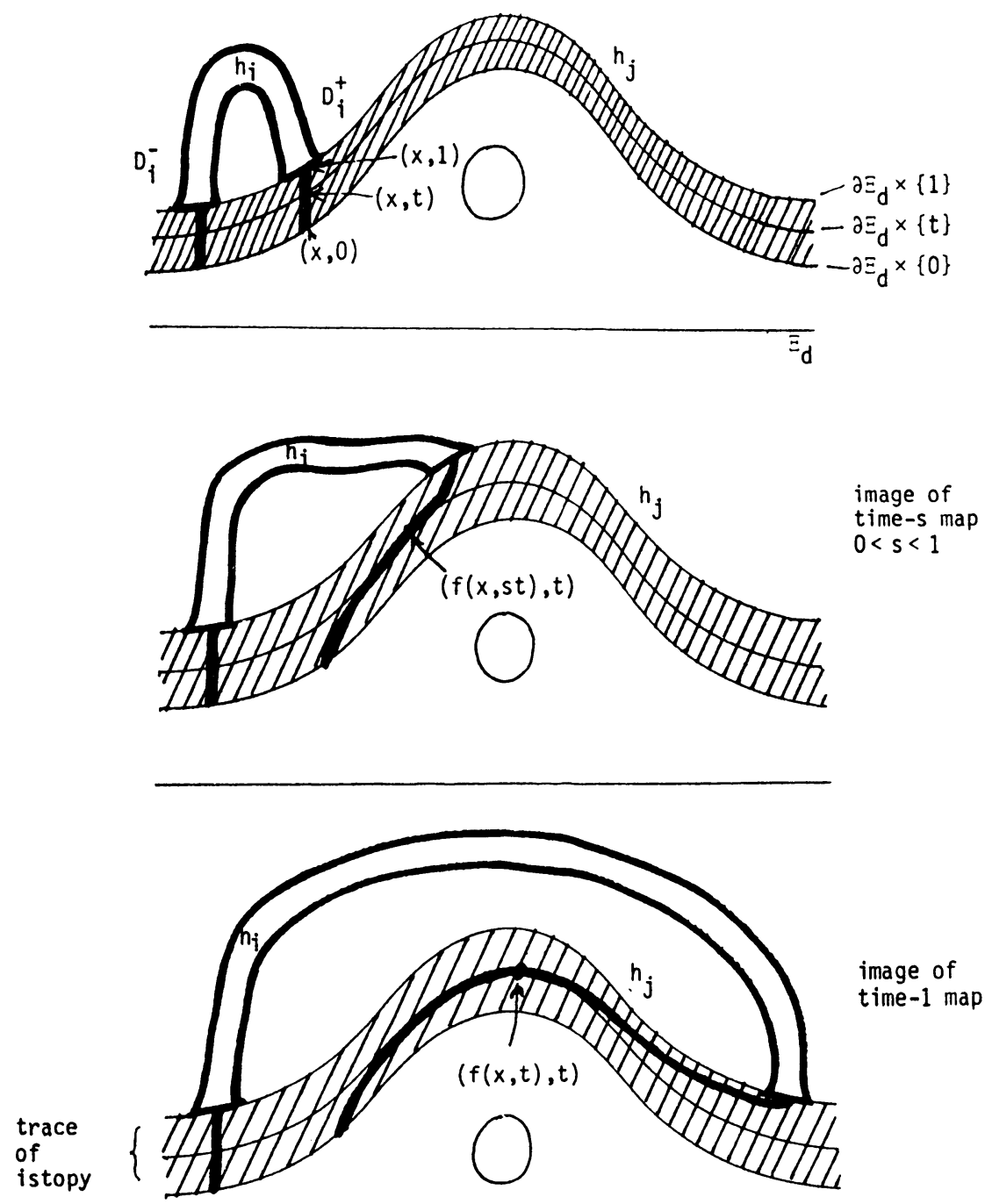

$f: \partial \Xi_{d} \times[0,1] \longrightarrow \partial \Xi_{d}:$ isotopy of $\partial \Xi_{d}$

FIGURE 3

$S^{3}$ as $R^{3} \cup\{\infty\}$. We then see $B \times\{-1\}$ as a neighbourhood of $\{\infty\}$ and $B \times\{1\}$ as the unit ball. To this we attach $d$-handles $H_{1}, \ldots, H_{d}$ where $H_{i} \cong h_{i} \times[-1,1]$, attached to $S^{3}$ along 3 -balls $B_{i}^{ \pm} \cong D_{i}^{ \pm} \times[-1,1]$ lying in $\partial B \times[-1,1]$.

Step 2 . To construct $M_{\phi} \cong \Xi_{d} \times_{\phi} S^{1}$ we identify $(x,-1)$ with $(\phi(x), 1), x \in \Xi_{d}$, in stages. Since $\left.\phi\right|_{B^{\prime}}$ is the identity map, we attach a 1 -handle $H_{*}$ to 3 -balls $B^{\prime} \times\{ \pm 1\}$. This has the effect of identifying part of the 1-spine of $\Xi_{d}$ with its image under $\phi$. To identify the remaining $d$ arcs with their images we attach 2 -handles $T_{i}, 1 \leq i \leq d$, 


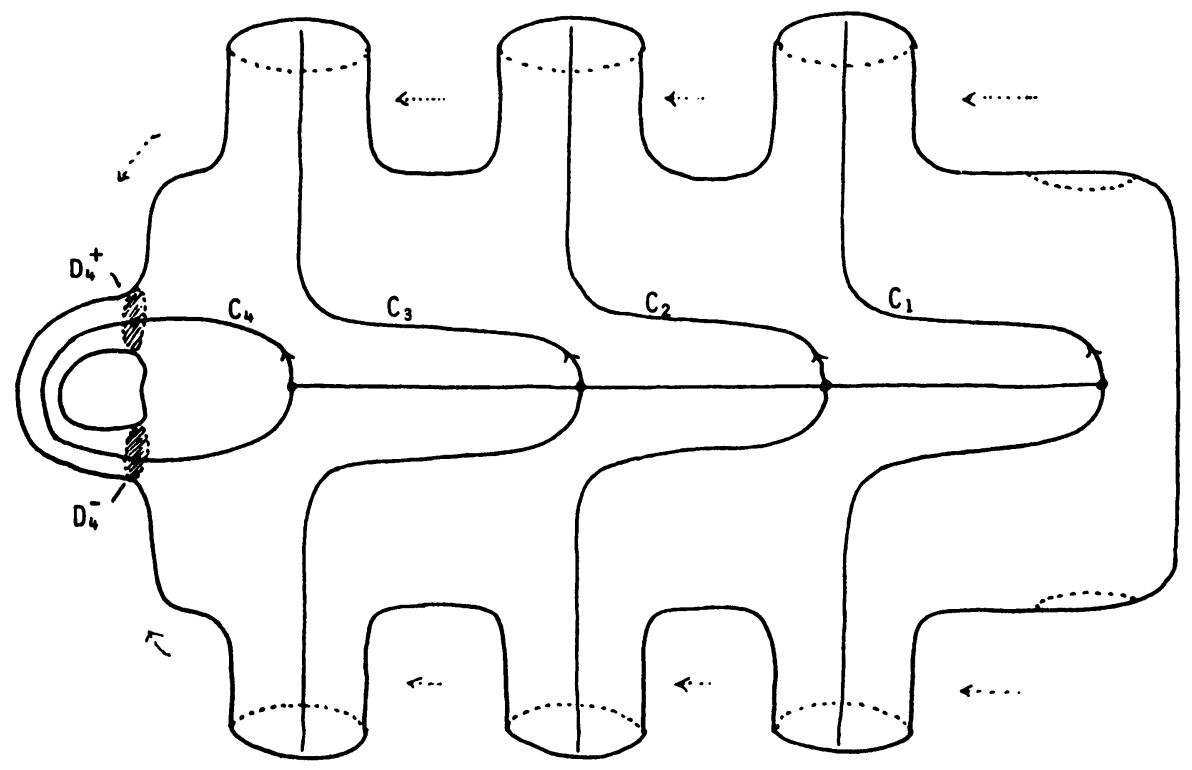

FIGURE 4

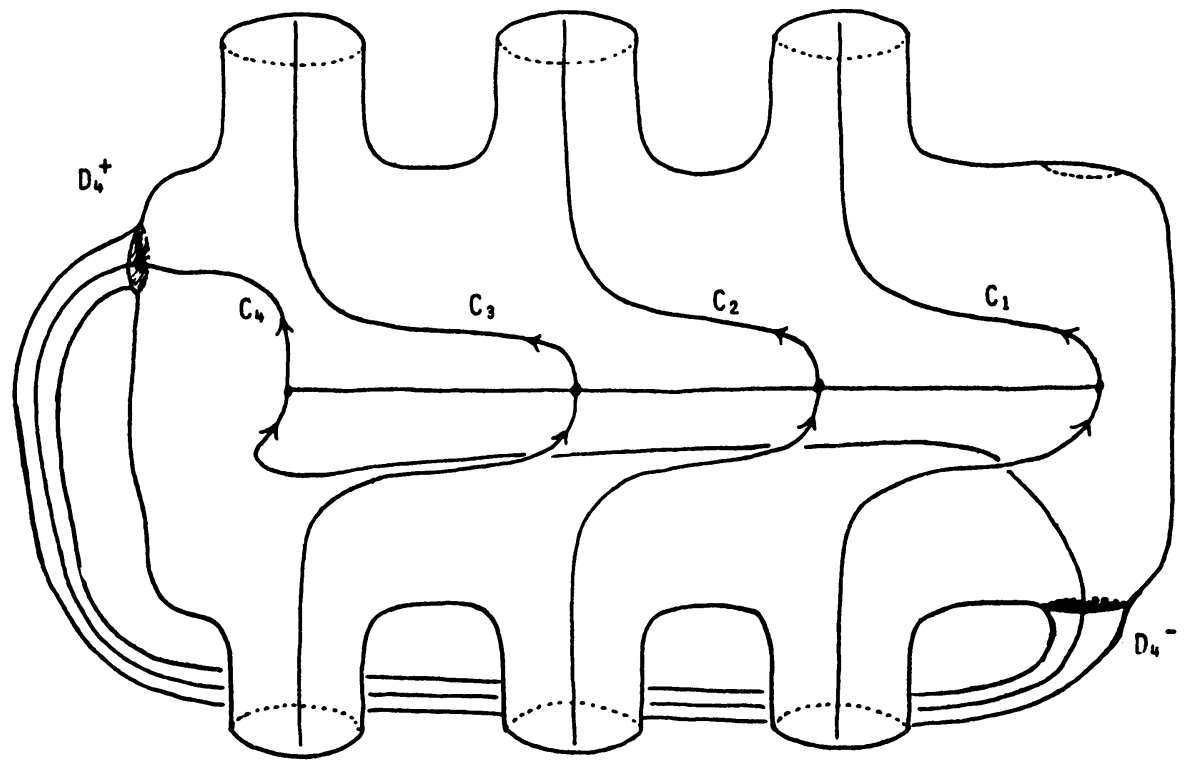

FIGURE 5

whose attaching circles and framings are determined by the annuli $A_{i}, 1 \leq i \leq d$, and their images under $\phi$. Notation is indicated in Figure 10 where, by abuse of notation, we denote by $T_{i}$ the attaching circles of the 2-handles $T_{i}$. Framings are determined by the dotted lines-- the numbers referring to the total number of twists of the framing annuli relative to parallel push-offs (in the plane of the page). It will be clear that the framing of $T_{d}$ need not be determined in order to know that 


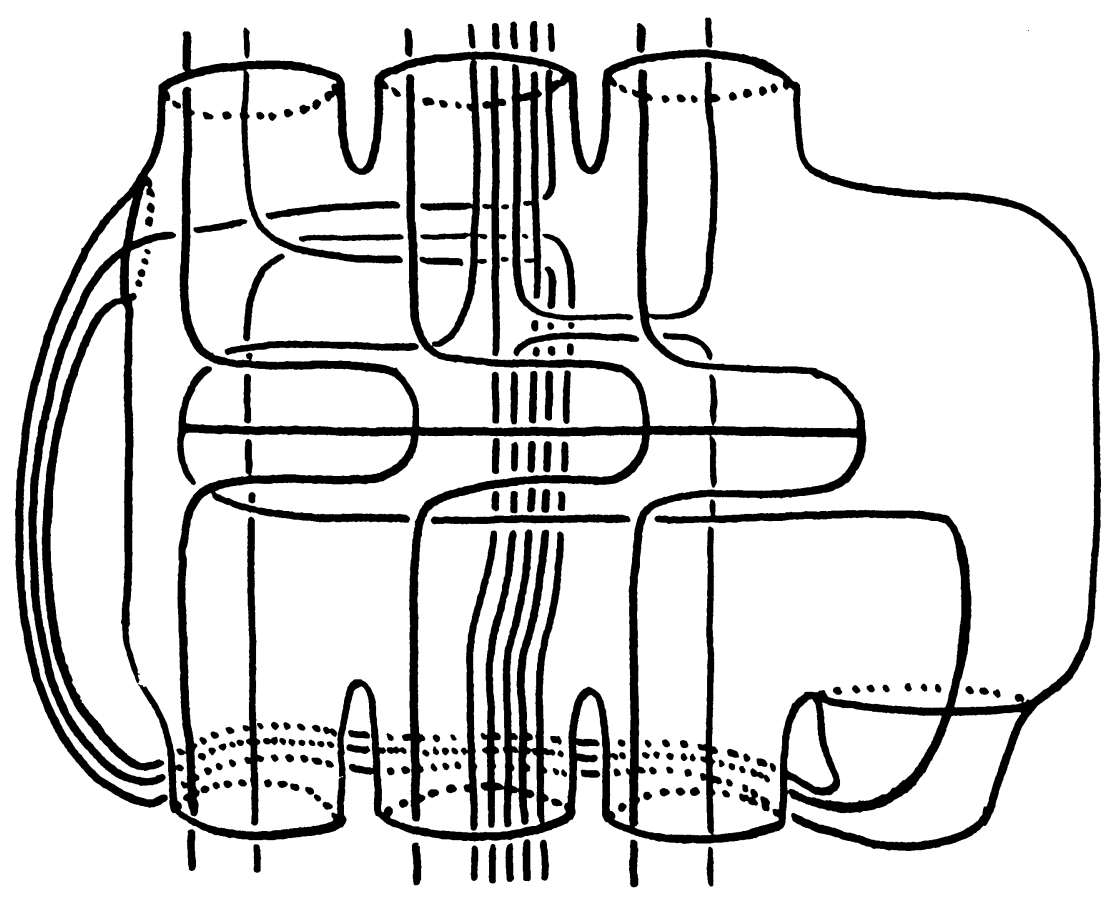

FIGURE 6

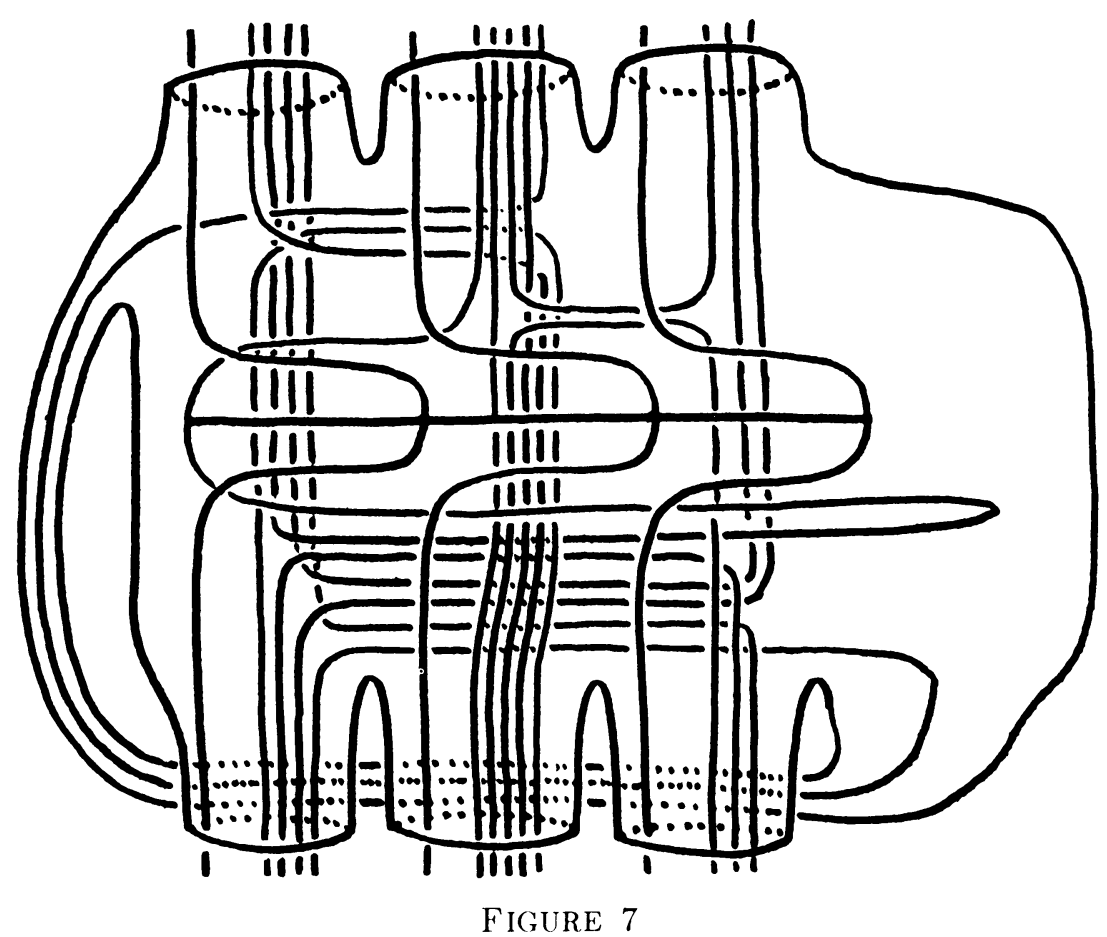




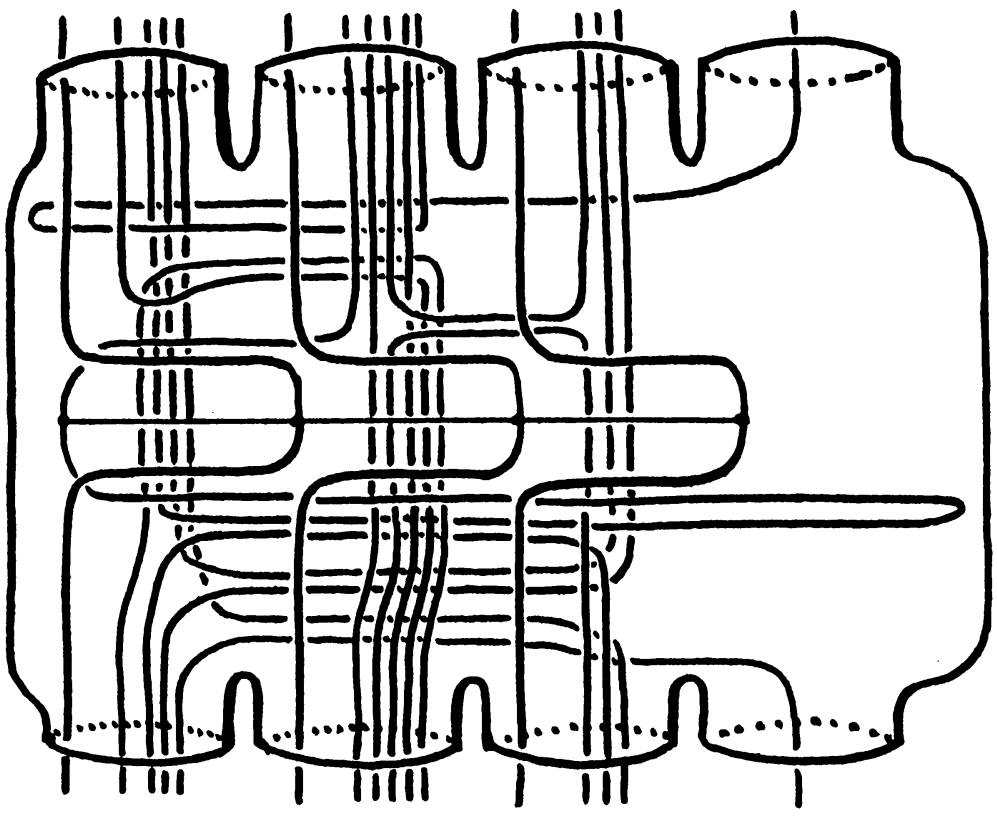

FIGURE 8

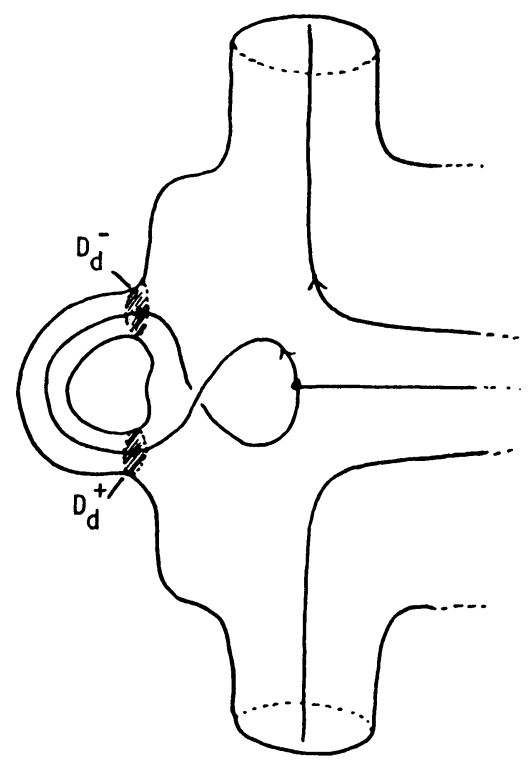

FIGURE 9

we have constructed a ribbon disc complement, but is important if wish to actually draw the ribbon knot with its ribbon.

It remains to be shown that $M_{\phi}$ is a ribbon disc complement in $B^{4}$. First we show that $B^{4}$ is obtained from $M_{\phi}$ by suitably attaching a 2-handle. (In fact, the ribbon disc will be the co-core of this 2-handle.) Attach the 2-handle with untwisted 


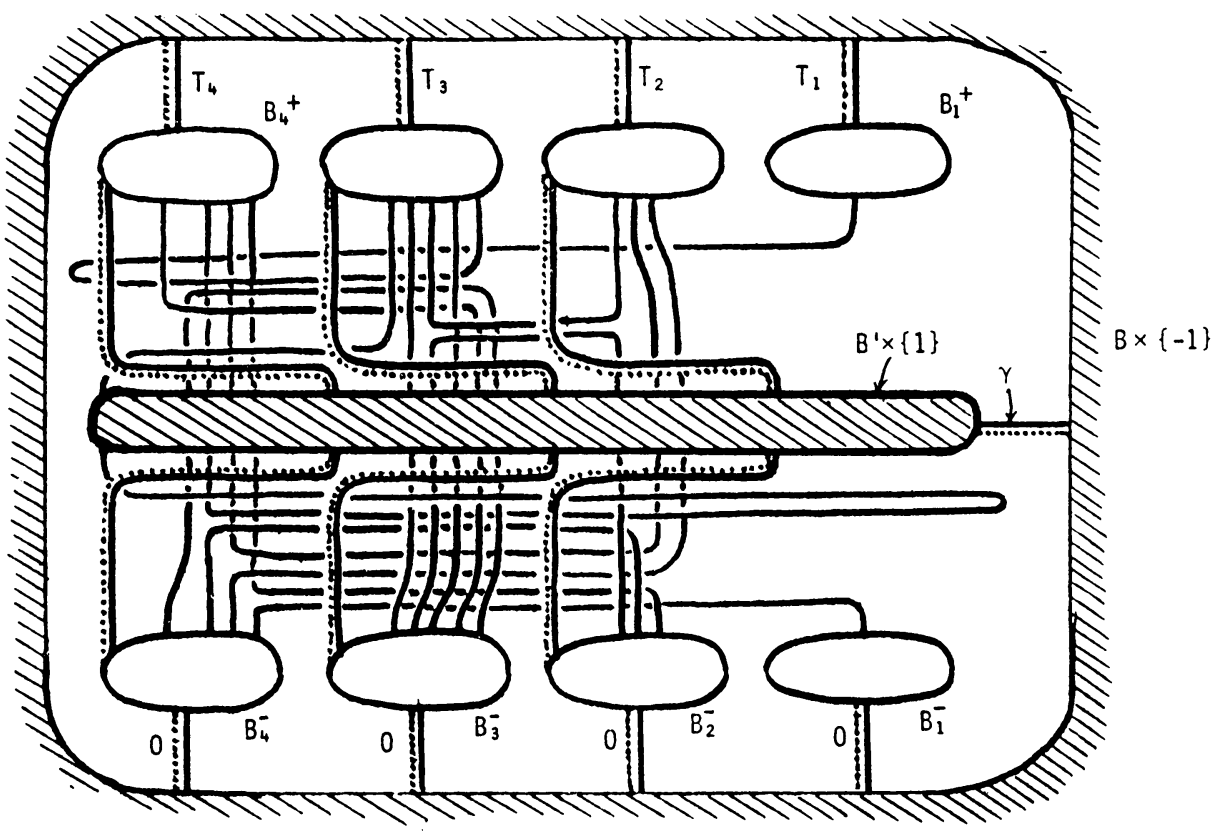

Figure 10

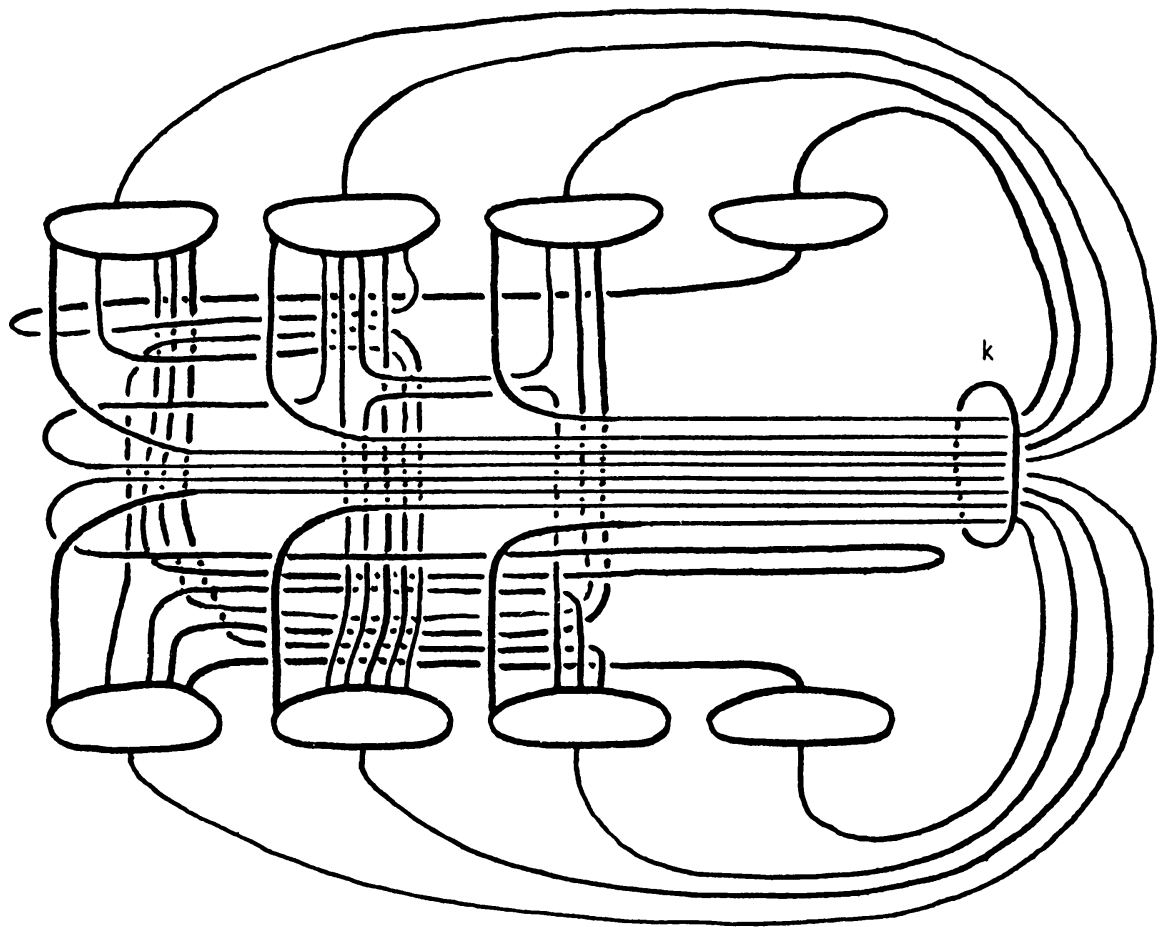

FIGURE 11 
framing along the circle represented by the arc $\gamma$ (Figure 10) whose endpoints are

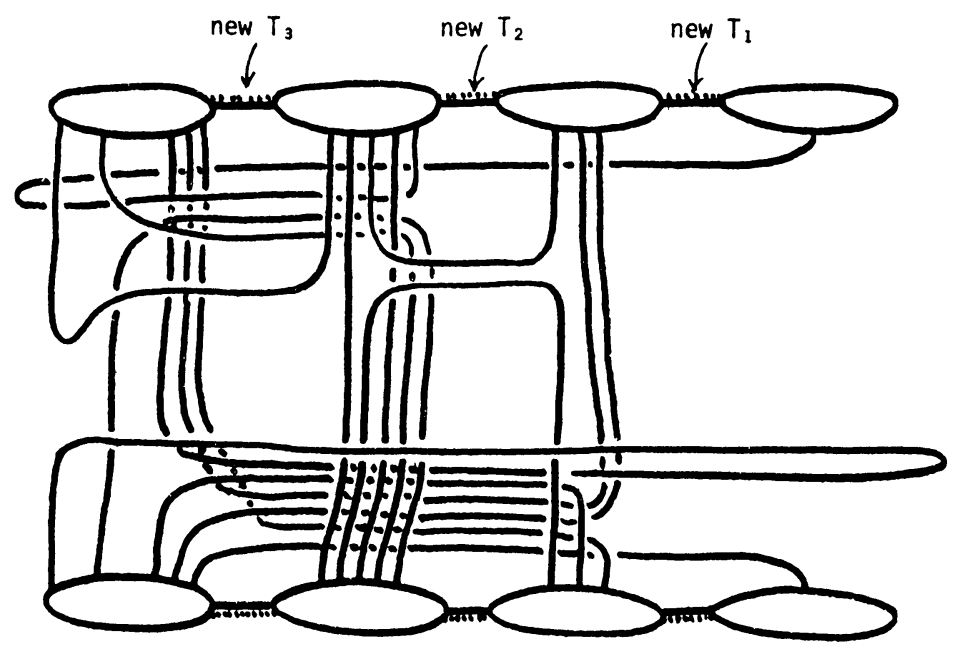

FIGURE 12

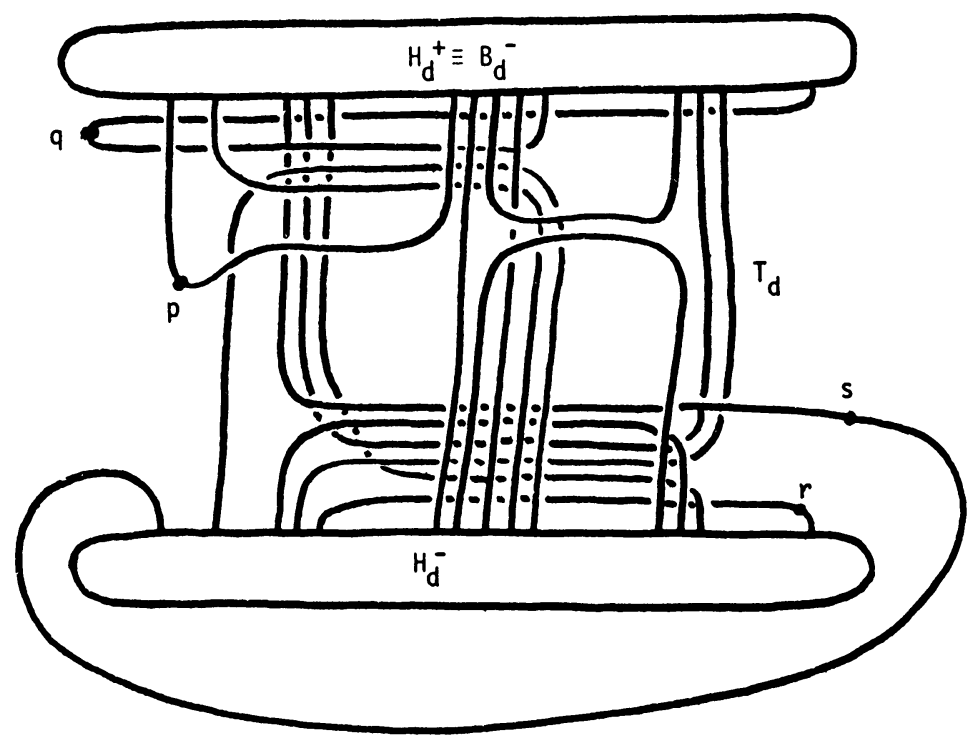

FIGURE 13

identified around the 1-handle $H_{*}$. Again, by abuse of notation, we refer to this 2-handle as $\gamma$. We see that the result is $B^{4}$ by sliding handles:

Slide all 2-handles $T_{1}, \ldots, T_{d}$ off $H_{*}$ using $\gamma$, and cancel $H_{*}$ with $\gamma$ (Figure 11). Rearranging the picture, we obtain Figure 12. Now cancel $H_{i}$ with $T_{i}$ consecutively for $i=1, \ldots, d-1$ to obtain Figure 13 .

We claim that the remaining pair of handles $T_{d}, H_{d}$ geometrically cancel. This is perhaps most easily seen by considering the arcs $p q$ and $r s$ of $T_{d}$ corresponding to the words $u$ and $v$, respectively. Since our construction of $\phi$ ensured that $c_{d}$ was 


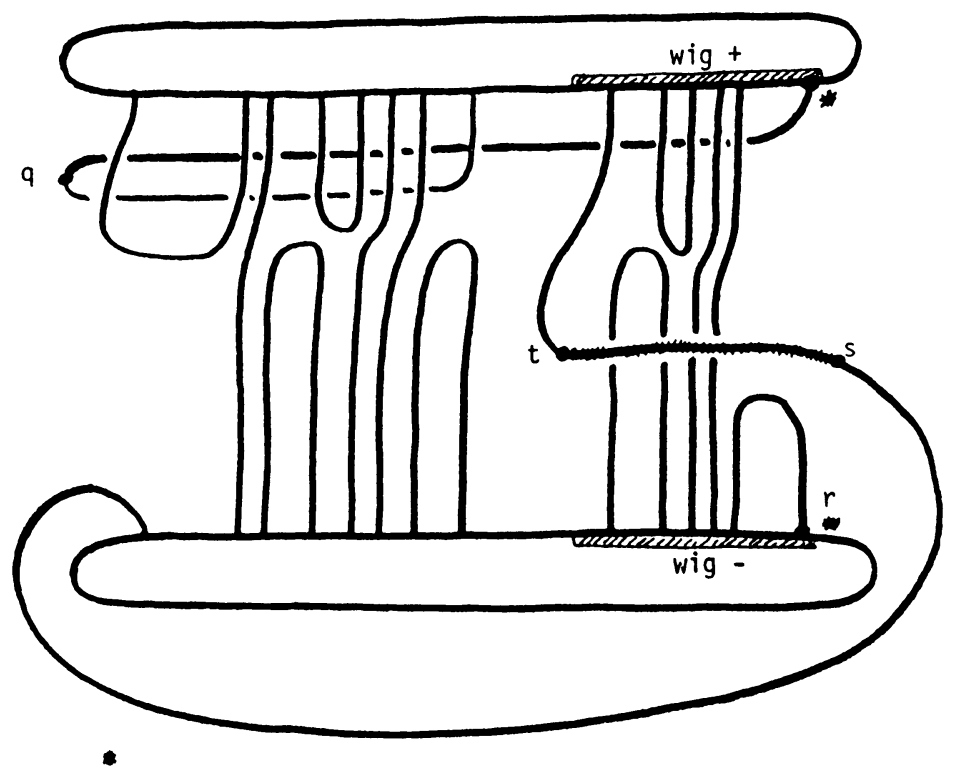

FIGURE 14

laid along inside $\Xi_{d}$, always travelling underneath what had been previously laid, both arcs may be isotoped into a vertical plane orthogonal to the page.

Now regard the arc st in Figure 14 as a "rope" and the arc $t r$ as part of an "iron gate", entering $H_{d}^{ \pm}$along the iron bars $w i g_{+}, w i g_{-}$and hinged at $*$. As we pull the rope up from the page, we swing the gate about its hinges, allowing it to swing out of the page and back on the other side of $*$, as in Figure 15. At this stage, the attaching circle for the 2-handle $T_{d}$ has only planar loops protruding from $H_{d}^{ \pm}$, and so by isotopy we can pull these loops over the 1-handle. Now $T_{d}$ travels over $H_{d}$ geometrically the same number of times as is computable algebraically. If $\sigma\left(\phi^{*}\right)=0$ or 2 , we obtain a cancelling pair and thus the result is $B^{4}$, as claimed.

REMARK. If $\sigma\left(\phi^{*}\right) \neq 0$ or 2 , we obtain (punctured lens space) $\times I$. This is the case in the illustrated example of $\S 3$.

To see the ribbon knot and ribbon disc, observe that the 2-handle $\gamma \cong D^{2} \times D^{2}$ is attached to $\Xi_{d} \times_{\phi} S^{1}$ along $D^{2} \times S^{1}$, where $D^{2} \subset \partial\left(\Xi_{d}\right) \cap \partial B^{\prime}$. The knot $k$ is $\partial D^{2} \times\{0\} \subset \partial D^{2} \times D^{2}$, and the disc $D$ is $D^{2} \times\{0\}$. It is easy to see that $\left(B_{0}, D\right)$ is diffeomorphic to the standard disc pair, where $B_{0}=B \times[-1,1] \cup H_{*} \cup \gamma$ (Figure 16). Note that the knot $k$ does not geometrically link any component of the attaching curves for $T_{1}, \ldots, T_{d-1}$ that are contained in $\partial B_{0}$. Consequently, $D$ remains a trivial disc knot in the 4-ball $B_{0} \cup H_{1} \cup \cdots \cup H_{d-1} \cup T_{1} \cup \cdots \cup T_{d-1}$. Pulling loops of $T_{d}$ over the 1-handle $H_{d}$ has the effect of isotoping "fingers" of $k$ over $H_{d}$ (Figure 17a). Using a collar neighbourhood, we isotope the disc $D$ as well. If the "fingers" are deformed slightly before isotopy, as in Figure 17b, the result is easily seen to be a ribbon disc in $B^{4}$. Notice that this deformation corresponds to the standard desingularization of an immersed ribbon disc in $S^{3}$.

It now follows that $k$ is a ribbon knot in $S^{3}$. In the general case it is complicated to describe $k$ and we have not done so; the knot $k$ is easily drawn for simple examples. In Figures $18-21$ we illustrate how $k$ must be isotoped in $H_{d}$ when we 


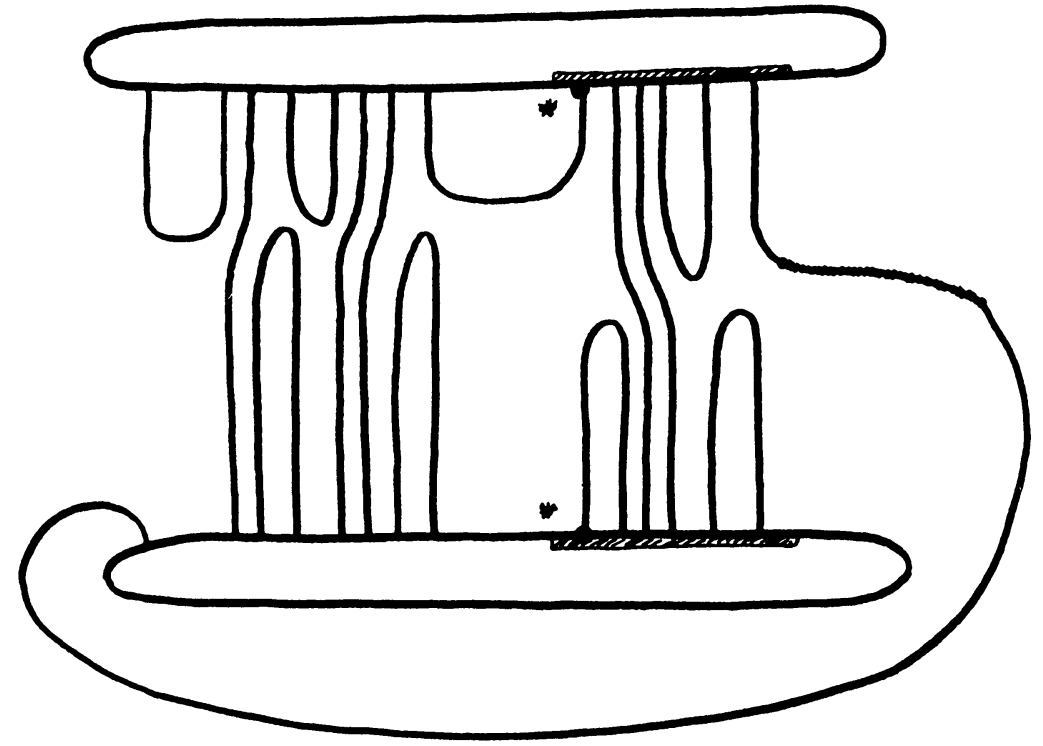

FIGURE 15

pull the loops of $T_{d}$ over the 1-handle $H_{d}$, for the case $d=2, \varepsilon=+1, u=\phi$, $v=x_{2}^{-1}$.

5. Invertibility of the ribbon disc pair. We show that having constructed $\phi$ by ambient isotopy in $S^{3}$, we may conclude that the ribbon disc pair $\left(B^{4}, D\right)$ is invertible.

Let $B^{\prime \prime}$ be a small 3-ball in $S^{3}-\operatorname{int}\left(\Xi_{d}\right)$ such that $B^{\prime \prime} \cap \partial\left(\Xi_{d}\right)=D$. By isotopy we may assume that $\left.\Phi\right|_{B^{\prime \prime}}$ is the identity map. Consider the mapping torus $\left(S^{3}-\right.$ int $\left.B^{\prime \prime}\right) \times_{\Phi} S^{1}$ which is diffeomorphic to $B^{3} \times S^{1}$. Observe that $D \subset$ $S^{2} \times S^{1}=\partial\left(B^{\prime \prime} \times S^{1}\right)$. Now regard $S^{2} \times D^{2}$ as $\left(D_{+}^{2} \times D^{2}\right) \cup\left(D_{-}^{2} \times D^{2}\right)$ and glue $S^{2} \times D^{2}$ to $B^{3} \times S^{1}$, first by attaching the 2-handle $D_{+}^{2} \times D^{2}=\gamma$ along $D_{+}^{2} \times S^{1}$, and then capping off with $D_{-}^{2} \times D^{2}$ to yield $S^{4}$. We obtain a trivial 2-knot $S^{2} \times\{0\} \subset S^{2} \times D^{2} \subset S^{4}$. Moreover, the intersection of $S^{2} \times\{0\}$ and $\left(\Xi_{d} \times_{\phi} S^{1}\right) \cup \gamma \cong B^{4}$ is equal to the ribbon disc $D$. Hence, $\left(B^{4}, D\right)$ is invertible.

6. Proof of Theorem 2. Let $C \subset S^{3} \times[0,1]$ be a ribbon concordance between $k_{0} \subset S^{3} \times\{0\}$ and $k_{1} \subset S^{3} \times\{1\}$. Recall that this means that $C$ is a concordance between $k_{0}$ and $k_{1}$ such that the restriction of the projection map $S^{3} \times I \rightarrow I$ is a Morse function with no local maxima (see [Go2]). Denote the exteriors of $k_{0}, k_{1}$ and $C$ by $X_{0}, X_{1}$ and $Y$, respectively. Let $\Delta_{k_{0}}, \Delta_{k_{1}}(t)$ and $\Delta_{C}(t)$ denote the Alexander polynomials of $\pi_{1}\left(X_{0}\right), \pi_{1}\left(X_{1}\right)$ and $\pi_{1}(Y)$, respectively. The proof of Theorem 2 will eventually follow from Theorem 1 together with the following result:

PROPOSITION 1. $\Delta_{k_{0}}(t) \Delta_{k_{1}}(t)=\Delta_{C}(t) \Delta_{C}\left(t^{-1}\right)$.

PrOOF. As in the proof of Lemma 3.2 of [Go2], we have $H_{1}(\tilde{Y}, \partial \tilde{Y} ; Q) \cong$ $H_{2}(\tilde{Y} ; Q) \simeq 0$. (Here denotes infinite cyclic cover, while $H_{*}(; Q)$ is regarded as a $\Lambda=Q\left[t, t^{-1}\right]$ module. The action of $t$ is induced, as usual, by the canonical 


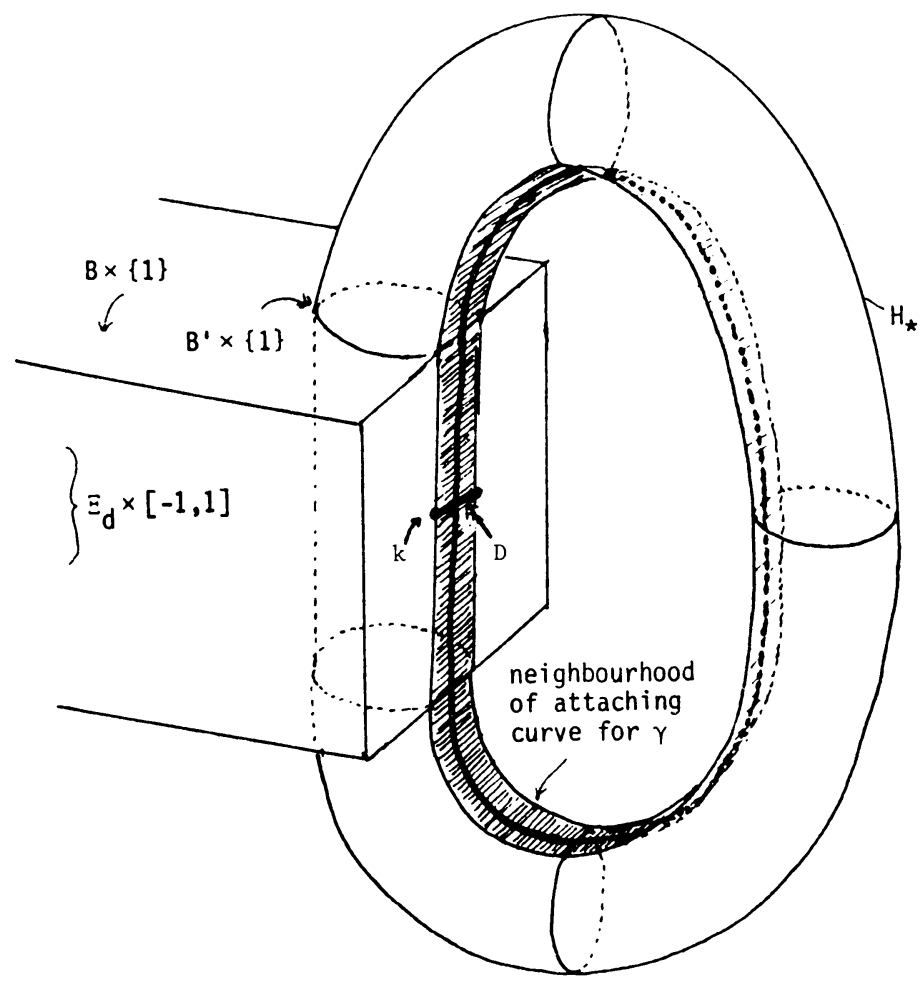

When the 2-handle $\gamma$ is attached, a trivial disc knot $D \subset B^{4}=B \times[-1,1] \cup H_{\star} \cup \gamma_{\star}$ is obtained.

FIGURE 16

generator of the group of covering transformations.) The inclusion map $\partial \tilde{Y} \rightarrow \tilde{Y}$ induces a short exact sequence

$$
0 \rightarrow H_{2}(\tilde{Y}, \partial \tilde{Y} ; Q) \rightarrow H_{1}(\partial \tilde{Y} ; Q) \rightarrow H_{1}(\tilde{Y} ; Q) \rightarrow 0 .
$$

Note that the $\Lambda$-module $H_{1}(\tilde{Y}: Q)$ has order $\left(\Delta_{C}(t)\right)$. By duality $[\mathbf{B l}, \mathbf{M i}]$ the module $H_{2}(\tilde{Y}, \partial \tilde{Y} ; Q)$ has order $\left(\Delta_{C}\left(t^{-1}\right)\right)$. Also, $H_{1}(\partial \tilde{Y} ; Q)$ is isomorphic to $H_{1}\left(\tilde{X}_{0} ; Q\right) \oplus H_{1}\left(\tilde{X}_{1} ; Q\right)$ and has order $\left(\Delta_{k_{0}}(t)\right)\left(\Delta_{k_{1}}(t)\right)$. However, order $H_{1}(\partial \tilde{Y} ; Q)$ is equal to (order $H_{2}(\tilde{Y}, \partial \tilde{Y} ; Q)$ ) (order $H_{1}(\tilde{Y} ; Q)$ ) using the short exact sequence above (see [Mi] for example). Consequently, the result claimed follows.

REMARK. Pat Gilmer has shown that $\Delta_{k_{1}}(t)=\Delta_{k_{0}}(t) f(t) f\left(t^{-1}\right)$ for some $f \in Z[t]$. (See [Gi, Proposition 1.4].) This formula arises in a pleasant manner from the above proposition. We are grateful to Professor Andrew Casson for suggesting the following argument.

The inclusion map $\tilde{X}_{1} \rightarrow \tilde{Y}$ induces a short exact sequence

$$
0 \rightarrow H_{2}\left(\tilde{Y}, \tilde{X}_{1} ; Q\right) \rightarrow H_{1}\left(\tilde{X}_{1} ; Q\right) \rightarrow H_{1}(\tilde{Y} ; Q) \rightarrow 0
$$



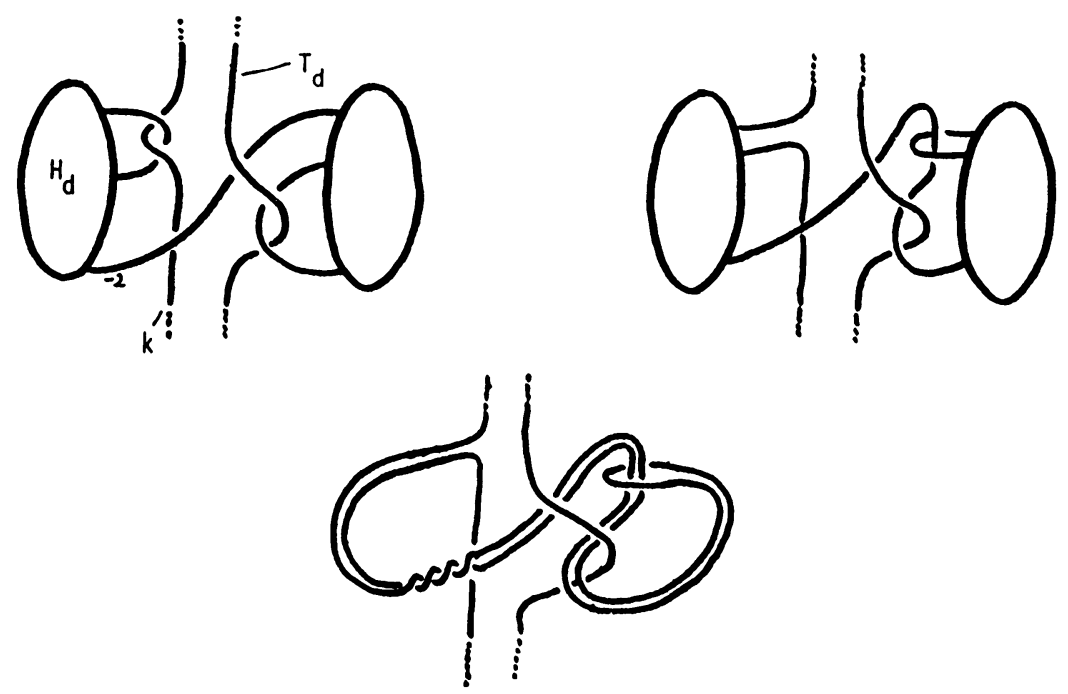

Equivalently:

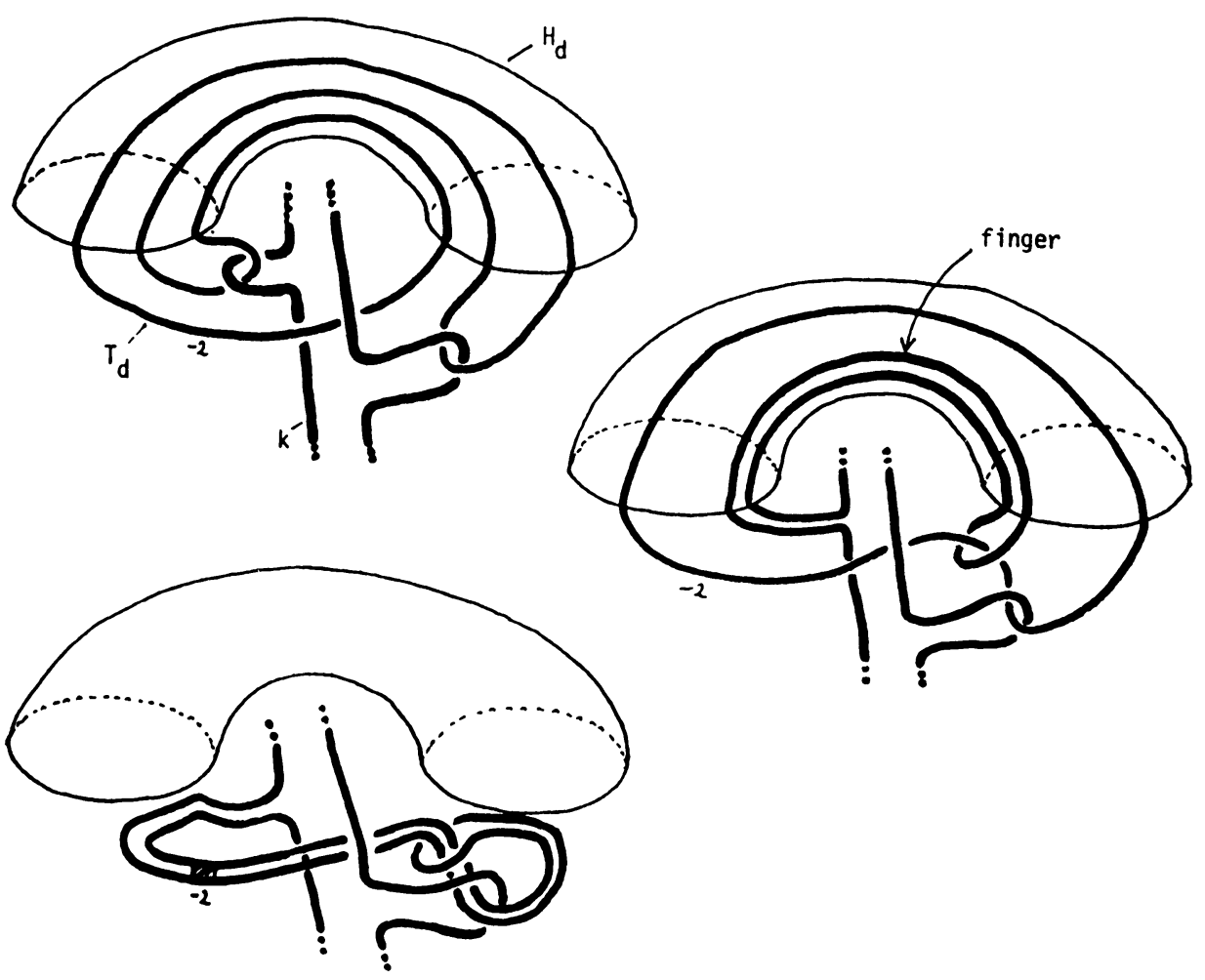

FIGURE17a 

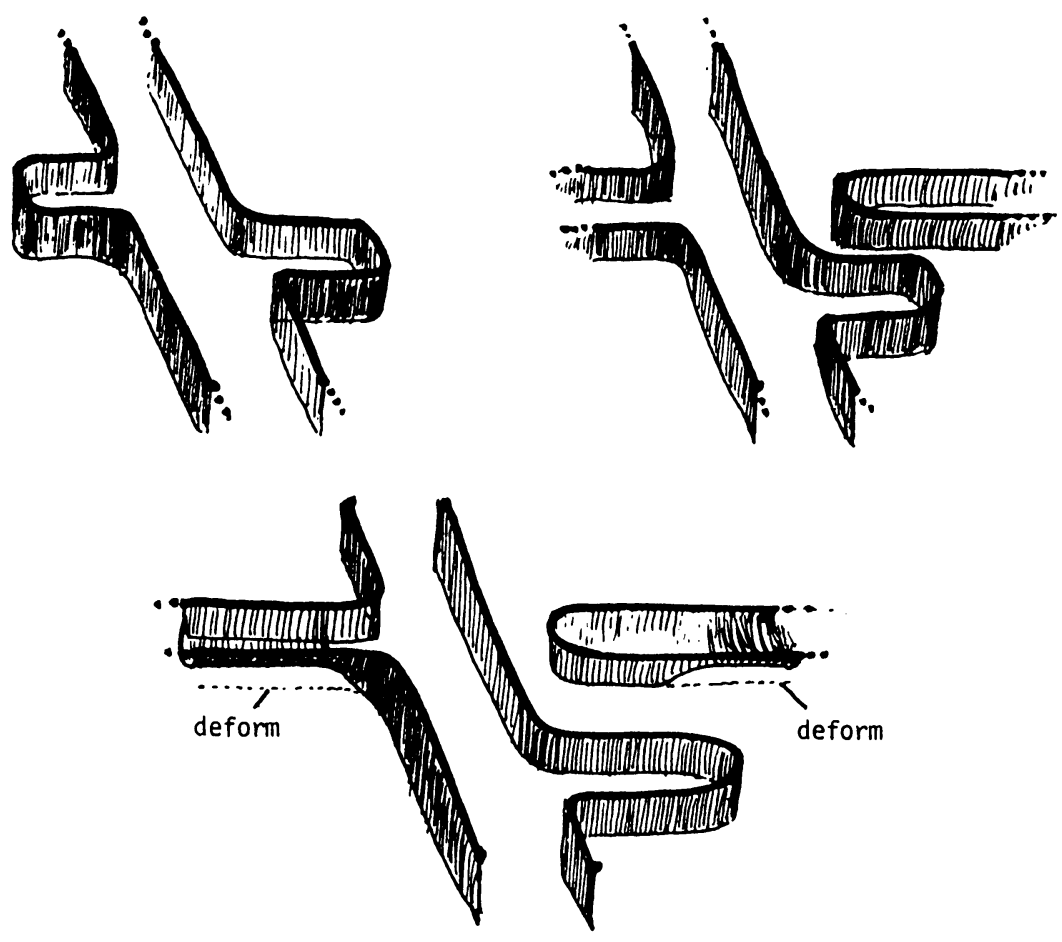

FIGURE $17 \mathrm{~B}$

Consequently, $\Delta_{k_{\mathrm{i}}}(t)=\Delta_{C}(t) f(t)$, where $f(t)$ generates order $\left(H_{2}\left(\tilde{Y}, \tilde{X}_{1} ; Q\right)\right)$. Since $\Delta_{k_{1}}(t)$ is symmetric, we also know that $\Delta_{k_{1}}(t)=\Delta_{C}\left(t^{-1}\right) f\left(t^{-1}\right)$. Now by Proposition 1 we have

$$
\left(\Delta_{k_{0}}(t) \Delta_{k_{1}}(t)\right) \Delta_{k_{1}}(t)=\left(\Delta_{C}(t) \Delta_{C}\left(t^{-1}\right)\right) \Delta_{k_{1}}(t)
$$

Substitution yields

$$
\Delta_{k_{0}}(t)\left(\Delta_{C}(t) f(t)\right) \Delta_{C}\left(t^{-1}\right) f\left(t^{-1}\right)=\Delta_{C}(t) \Delta_{C}\left(t^{-1}\right) \Delta_{k_{1}}(t) .
$$

Cancellation now reveals the desired formula.

We now prove Theorem 2. Let $\phi^{*}$ be the automorphism of the free group $F$ defined by

$$
\phi^{*}\left(x_{i}\right)= \begin{cases}x_{i+1} & \text { if } 1 \leq i<d, \\ \left(x_{1}^{a_{0}} x_{2}^{a_{1}} \cdots x_{d}^{a_{d-1}}\right)^{-a_{d}} & \text { if } i=d .\end{cases}
$$

(Compare with [As-Yo, p. 268].) By Theorem 1 there exists a fibred ribbon disc $\left(B^{4}, D\right)$ with monodromy $\phi^{*}$. Recall that $\phi^{*}$ induces an automorphism $\alpha$ of the free abelian group $F / F^{\prime}$, and the Alexander polynomial of $\pi_{1}\left(B^{4}, D\right)$ is equal to $\operatorname{det}(\alpha-t I)$. An easy calculation shows that the Alexander polynomial is, in fact, equal to $f(t)$. Let $k=\partial D \subset S^{3}$. By Proposition 1 (with $k_{0}$ the unknot) the Alexander polynomial of $k$ is equal to $f(t) f\left(t^{-1}\right)$. Moreover, $k$ is doubly slice since $\left(B^{4}, D\right)$ is invertible.

REMARKS. 1. In [As-Yo] Asano and Yoshikawa proved that any integral polynomial $f(t)=a_{0}+a_{1} t+\cdots+a_{d} t^{d}$ with $f(1)= \pm 1$ and $a_{0} a_{d}= \pm 1$ is the Alexander 


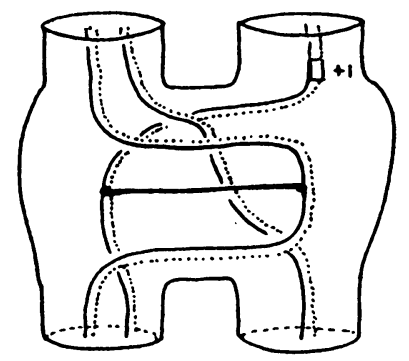

FIGURE 18
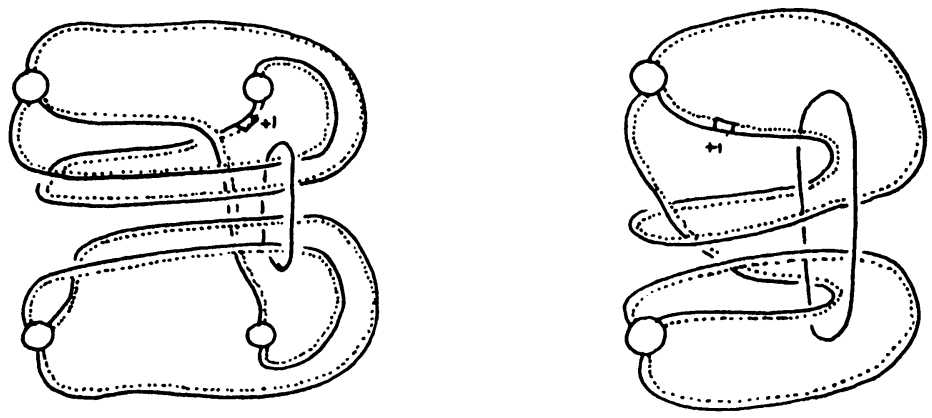

FIGURE 19

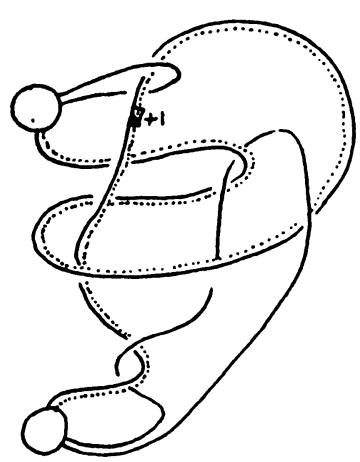

FIGURE 20

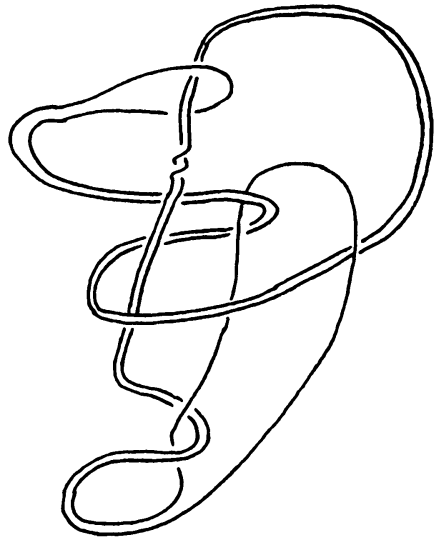

FIGURE 21

polynomial of a fibred 2-knot in $S^{4}$. Theorem 2 can be regarded as a 4 -dimensional analog of this result. It now follows that each fibred 2-knot constructed in [As-Yo] is, in fact, the double of some invertible fibred ribbon disc.

2. Since $f(t)$ arises from the Abelianisation of the monodromy, it is possible that we can construct either infinitely many knots with the same Alexander polynomial, for each $f(t)$, or infinitely many distinct ribbons for the same knot (since the complement of the ribbon disc in $B^{4}$ has one-relator fundamental group, and different relations arise by choosing $u$ and $v$ differently), or both. We hope to investigate this in a later paper. 
7. The genus 2 case. We can now realise all possible Seifert matrices for genus 2 fibred doubly slice ribbon knots by knots having these geometric properties.

PROPOSITION 2. There are exactly three $S$-equivalence classes of genus 2 fibred doubly slice knots in $S^{3}$. Moreover, each class can be represented by a fibred ribbon doubly slice knot.

REMARK. The reader may consult [Go1] for the definition of $S$-equivalence.

PROOF. Let $k$ be any genus 2 fibred doubly slice knot in $S^{3}$. It is well known that such a knot admits a Seifert matrix of type $\left(\begin{array}{cc}0 & A \\ B & 0\end{array}\right)$ where $A$ and $B$ are unimodular (i.e. contained in $G L(2 ; Z)$ ). We may apply the congruence

$$
\left(\begin{array}{cc}
1 & 0 \\
0 & B^{-1}
\end{array}\right)\left(\begin{array}{cc}
0 & A \\
B & 0
\end{array}\right)\left(\begin{array}{cc}
1 & 0 \\
0 & B^{-t}
\end{array}\right)=\left(\begin{array}{cc}
0 & A B^{-t} \\
1 & 0
\end{array}\right)
$$

in order to find an equivalent matrix. Consequently, such a knot has a Seifert matrix of type $\left(\begin{array}{cc}0 & P \\ 1 & 0\end{array}\right)$ where $P \in G L(2 ; Z)$.

Observe that if $Q$ is any unimodular matrix that is similar to $P$, the two matrices $\left(\begin{array}{ll}0 & P \\ 1 & 0\end{array}\right)$ and $\left(\begin{array}{ll}0 & Q \\ 1 & 0\end{array}\right)$ are congruent and hence $S$-equivalent. Specifically, if $X^{-1} P X=$ $Q$, then

$$
\left(\begin{array}{cc}
X & 0 \\
0 & X^{-t}
\end{array}\right)\left(\begin{array}{cc}
0 & Q \\
1 & 0
\end{array}\right)\left(\begin{array}{cc}
X^{t} & 0 \\
0 & X^{-1}
\end{array}\right)=\left(\begin{array}{cc}
0 & P \\
1 & 0
\end{array}\right) .
$$

Note also that the Alexander polynomial $\Delta_{k}(t)$ of $k$ is given by $\Delta_{k}(t)=$ $\operatorname{det}(t I-P) \operatorname{det}\left(t I-P^{-t}\right)$. Since $\Delta_{k}(1)= \pm 1$, it follows that $I-P$ is also unimodular. It is known that there are exactly four similarity classes of matrices $P \in G L(2 ; Z)$ such that $I-P$ is also unimodular (see [Ta or Ra1]. The four classes are distinguished by their characteristic polynomials, and are represented by

$$
P_{1}=\left(\begin{array}{cc}
0 & -1 \\
1 & 1
\end{array}\right), \quad P_{2}=\left(\begin{array}{cc}
0 & -1 \\
1 & 3
\end{array}\right), \quad P_{3}=\left(\begin{array}{cc}
0 & 1 \\
1 & 1
\end{array}\right), \quad P_{4}=\left(\begin{array}{cc}
0 & 1 \\
1 & -1
\end{array}\right)
$$

Consequently we need only consider the following potential Seifert matrices for $k$ :

$$
S_{i}=\left(\begin{array}{cc}
0 & P_{i} \\
1 & 0
\end{array}\right), \quad i=1,2,3,4
$$

Notice that $S_{3}$ and $S_{4}$ are congruent: letting

$$
C=\left(\begin{array}{cc|cc}
0 & 0 & 1 \\
& & 1 & 0 \\
\hline 1 & 0 & 0 \\
1 & 1 & 0
\end{array}\right)
$$

we see that $C S_{4} C^{t}=S_{3}$. Hence we need only consider $S_{1}, S_{2}$ and $S_{3}$.

An easy calculation produces the corresponding Alexander polynomial $\Delta_{i}(t)$ for $S_{i}$ :

$$
\begin{aligned}
& \Delta_{1}(t)=\left(t^{2}-t+1\right)\left(t^{-2}-t^{-1}+1\right)=t^{2}-2 t+3-2 t^{-1}+t^{-2} \\
& \Delta_{2}(t)=\left(t^{2}-3 t+1\right)\left(t^{-2}-3 t^{-1}+1\right)=t^{2}-6 t+11-6 t^{-1}+t^{-2} \\
& \Delta_{3}(t)=\left(t^{2}-t-1\right)\left(t^{-2}-t^{-1}-1\right)=t^{2}-3+t^{-2}
\end{aligned}
$$



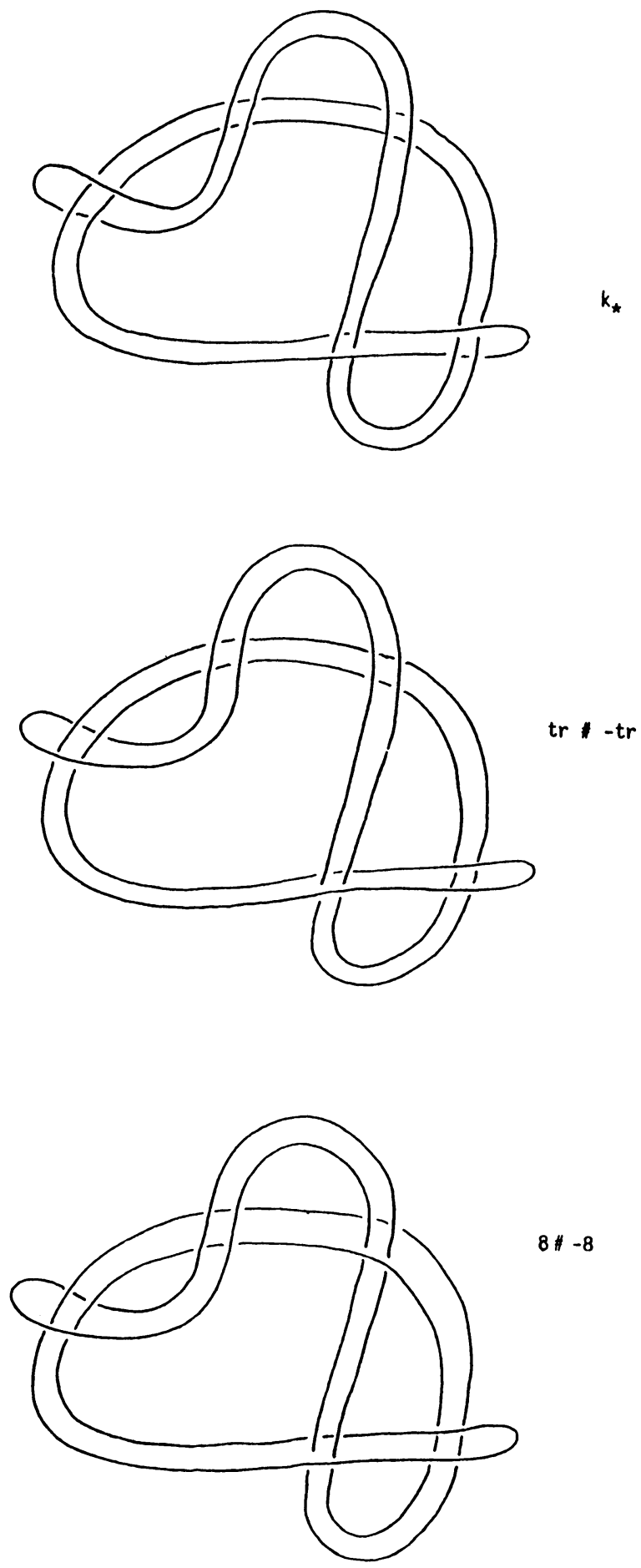

FIGURE 22 
Since the Alexander polynomial is an invariant of $S$-equivalence, the matrices $S_{1}, S_{2}$ and $S_{3}$ represent distinct classes.

To complete the proof, we realise each of these classes by a knot. For $S_{1}$ we take $3_{1} \#\left(-3_{1}\right)$, for $S_{2}$ we take $4_{1} \#\left(-4_{1}\right)$, and for $S_{3}$ we take the knot $k_{*}$ of Figure 22 . This last knot has been constructed as in Theorem 2 with the desired Alexander polynomial and hence with the desired Seifert matrix. It is not difficult to see that $k_{*}$ can in fact be drawn with only 11 crossings.

In the genus 2 case it is easy to give all possible knots arising from the construction: the words $u$ and $v$ can only be powers of $x_{2}$, and thus we are led to 4 possible classes for $w$ :

(a) $w=x_{2}^{k} x_{1} x_{2}^{-1-k}$

(b) $w=x_{2}^{k} x_{1} x_{2}^{1-k}$,

(c) $w=x_{2}^{k} x_{1}^{-1} x_{2}^{1-k}$,

(d) $w=x_{2}^{k} x_{1}^{-1} x_{2}^{3-k}$.

For $f(t)$ the polynomial of the trefoil or figure $8 \mathrm{knot}$, it would be of interest to compare the knots with those constructed in $[\mathbf{A i}, \mathbf{K a}, \mathbf{Q u}-\mathbf{W e}$ and $\mathbf{S t}]$.

8. Questions. 1. Does Theorem 1 generalise to a larger class of diffeomorphisms?

2. Can all possible Seifert matrices be realised geometrically as the matrices corresponding to knots arising as the boundaries of invertible fibred ribbon discs?

\section{Appendix: On a theorem of Yoshikawa.}

1. Introduction. Let $K \subset S^{4}$ be a ribbon 2 -knot of 1 fusion. Denote the fundamental group $\pi_{1}\left(S^{4}-K\right)$ by $G$. In [Yo] Yoshikawa has shown that $K$ is fibred iff the commutator subgroup $G^{\prime}$ is finitely generated. We sharpen this result as follows:

THEOREM. Let $K \subset S^{4}$ be a ribbon 2 -knot of 1 fusion. Then $\left(S^{4}, K\right)$ is the double of some invertible fibred ribbon disc pair iff $G^{\prime}$ is finitely generated.

REMARK. If $G^{\prime}$ is finitely generated, then $G^{\prime}$ is free by [Ra2].

For the definitions of terms used above, the reader may consult $[\mathbf{M a}, \mathbf{Y a}, \mathbf{S u}$ or Ai-Si], for example.

2. An algebraic lemma. If $w$ is a word in $n$ letters $x_{1}, \ldots, x_{n}$, then $\sigma\left(w ; x_{i}\right)$ will denote the exponent sum of $x_{i}$ in $w$. The following result is essentially contained in [Yo].

LEMMA. Let $G$ be a group with 1-relator presentation $P=\left(x, a_{1} ; r\right)$, where $\sigma(r ; x)=0$ and $\sigma\left(r ; a_{1}\right)= \pm 1$. Assume that the commutator subgroup $G^{\prime}$ is finitely generated. Then $P$ is $A C$-equivalent to a presentation

$$
\tilde{P}=\left(x, a_{1}, \ldots, a_{d} ; x a_{i} x^{-1} a_{i+1}^{-1}, 1 \leq i<d, x a_{d} x^{-1} w^{-1}\right)
$$

where $d$ is the degree of the Alexander polynomial of $G$ and $w$ is a word in $a_{1}, \ldots, a_{d}$. Moreover, $a_{1}^{ \pm 1}$ occurs exactly once in $w$.

For the definition of $A C$-equivalence the reader may consult [ $\mathbf{A n - C u}, \mathbf{L e}$ or $\mathbf{Y o}$ ].

PROOF OF LEMMA The first assertion is proved in [Yo]. We need only show that $a_{1}^{ \pm 1}$ occurs exactly once in $w$. By [Ra2] (see Lemma 5) the map $a_{1} \rightarrow$ $a_{2}, \ldots, a_{d-1} \rightarrow a_{d}, a_{d} \rightarrow w$ determines an automorphism of the free group $F\left(\cong G^{\prime}\right)$ 
of rank $d$. Consequently, $a_{2}, \ldots, a_{d-1}, w$ is a basis for $F$. Applying elementary Nielsen transformations (see [MKS]) we may assume without loss of generality that the first and last letters (not necessarily the same) of $w$ are $a_{1}^{ \pm 1}$. Then words in $a_{2}, \ldots, a_{d}, w$ produce no nontrivial cancellation except that which may occur in powers of $w$. Notice that for each $k \neq 0, w^{k}$ contains at least as many occurrences of $a_{1}^{ \pm 1}$ as does $w$. (To see this, write $w$ as $u v u^{-1}$, where $v$ is cyclically reduced.) Since $a_{1}$ can be expressed as a word in $a_{2}, \ldots, a_{d}, w$, it follows that $w$ is equal to $a_{1}^{ \pm 1}$.

3. Proof of theorem. Assume that $\left(S^{4}, K\right)$ is the double of a ribbon disc pair $\left(B^{4}, D\right)$. The inclusion map $\left(S^{3}-\partial D\right) \rightarrow\left(B^{4}-D\right)$ induces a surjection on fundamental groups. By van Kampen's Theorem the inclusion map $\left(B^{4}-D\right) \rightarrow$ $\left(S^{4}-K\right)$ induces an isomorphism $\pi_{1}\left(B^{4}-D\right) \cong G$. Consequently, if $\left(B^{4}, D\right)$ is fibered then $G^{\prime}$ is finitely generated. (See [Ke-We] for example.)

Conversely, assume that $G^{\prime}$ is finitely generated. Since $K$ is a ribbon 2-knot of 1 fusion, $\left(S^{4}, K\right)$ is the boundary of a disc pair $\left(B^{5}, B^{3}\right)$ with the following properties (see $[\mathbf{H i}])$ :

Let $A$ denote $\operatorname{cl}\left(B^{5}-\operatorname{nhd}\left(B^{3}\right)\right)$, where "cl" denotes closure and "nhd" denotes neighbourhood. Then

(i) $A$ has a handle decomposition $H^{0} \cup H^{1} \cup H_{1}^{1} \cup H_{1}^{2}$, where $H_{*}^{k}$ denotes a $k$-handle.

(ii) $B^{5}=A \cup H^{2}$, where $H^{2}$ is a suitably attached 2-handle that cancels $H^{1}$. (Note that $B^{3}$ is then the cocore of $H^{2}$.)

Let $x, a_{1}$ denote the free generators of $\pi_{1}\left(H^{0} \cup H^{1} \cup H_{1}^{1}\right)$ corresponding to $H^{1}, H_{1}^{1}$, respectively. Then $\pi_{1}(A)$ has an associated presentation $P=\left(x, a_{1} ; r\right)$, where $r$ is a word in $x, a_{1}$. Clearly $\sigma\left(r ; a_{1}\right)= \pm 1$. By sliding $H_{1}^{1}$ over $H^{1}$, we may assume without loss of generality that $\sigma(r ; x)=0$. (See [Hi, Theorem 5.1].) By the above lemma, $P$ is $A C$-equivalent to a presentation

$$
\tilde{P}=\left(x, a_{1}, \ldots, a_{d} ; x a_{i} x^{-1}=\phi^{*}\left(a_{i}\right), 1 \leq i \leq d\right)
$$

with $\phi^{*}$ an automorphism of $G^{\prime}(\cong F)$ of form:

$$
\phi^{*}\left(a_{i}\right)= \begin{cases}a_{i+1} & \text { if } 1 \leq i<d, \\ w=u a_{1}^{\varepsilon} v^{-1} & \text { if } i=d(\varepsilon= \pm 1),\end{cases}
$$

where $u$ and $v$ are arbitrary words (possibly empty) in $F$ not containing $a_{1}^{ \pm 1}$.

By $[\mathbf{A n}-\mathbf{C u}]$ the manifold $A$ has a handle decomposition

$$
A \cong H^{0} \cup H^{1} \cup H_{1}^{1} \cup \cdots \cup H_{d}^{1} \cup H_{1}^{2} \cup \cdots \cup H_{d}^{2}
$$

corresponding to the presentation $\tilde{P}$.

We proved in $[\mathbf{A i}-\mathbf{S i}]$ that there exists an invertible fibred disc pair $\left(B^{4}, D\right)$ with fibre a genus $d$ handlebody and monodromy $\phi^{*}$. Consider the disc pair $\left(B^{4} \times I\right.$, $D \times I)$, which we will denote by $\left(\tilde{B}^{5}, \tilde{B}^{3}\right)$. We will prove that $\left(\tilde{B}^{5}, \tilde{B}^{3}\right)$ is diffeomorphic to $\left(B^{5}, B^{3}\right)$. Since the boundary of $\left(\tilde{B}^{5}, \tilde{B}^{3}\right)$ is the double of an invertible fibred ribbon disc pair, the proof of the theorem will be complete.

In $[\mathbf{A} \mathbf{i}-\mathbf{S i}]$ we used techniques of Akbulut and Kirby [Ak-Ki] to describe an explicit handle decomposition for $\operatorname{cl}\left(B^{4}-\operatorname{nhd}(D)\right)$, consisting only of 0-, 1- and 
2-handles, corresponding to the presentation $\tilde{P}$. The ribbon disc $D$ was seen as the cocore of an additional 2-handle $\gamma$ such that $\gamma \cup \operatorname{cl}\left(B^{4}-\operatorname{nhd}(D)\right) \cong B^{4}$.

Hence $\tilde{A}=\operatorname{cl}\left(\tilde{B}^{5}-\operatorname{nhd}\left(\tilde{B}^{3}\right)\right)$ acquires a product handle decomposition $\tilde{H}^{0} \cup$ $\tilde{H}^{1} \cup \tilde{H}_{1}^{1} \cup \cdots \cup \tilde{H}_{d}^{1} \cup \tilde{H}_{1}^{2} \cup \cdots \cup \tilde{H}_{d}^{2}$. Moreover, $\tilde{H}^{2} \cong \gamma \times I$ is a 2-handle that cancels $\tilde{H}^{1}$, and $\tilde{B}^{3}$ is the cocore. Identify $H^{0} \cup H^{1} \cup H_{1}^{1} \cup \cdots \cup H_{d}^{1} \subset A$ with $\tilde{H}^{0} \cup \tilde{H}^{1} \cup \tilde{H}_{1}^{1} \cup \cdots \cup \tilde{H}_{d}^{1} \subset \tilde{A}$. By isotopy we may assume that the attaching circle of $\tilde{H}^{2}$ (resp. $\left.\tilde{H}_{i}^{2}, 1 \leq i \leq d\right)$ is the same as that of $H^{2}$ (resp. $\left.H_{i}^{2}, 1 \leq i \leq d\right)$. Observe that for each 2-handle in the decomposition of $\tilde{B}^{5}$ there corresponds a 1 handle over which it travels algebraically \pm 1 times (while travelling over the other 1-handles algebraically 0 times). Consequently we can cut, twist and reglue each of the 1-handles in $\tilde{B}^{5}$, if necessary, so that the framing of $\tilde{H}^{2}$ (resp. $\tilde{H}_{i}^{2}, 1 \leq i \leq d$ ) is the same as for $H^{2}$ (resp. $\left.H_{i}^{2}, 1 \leq i \leq d\right)$. It is now apparent that $\left(\tilde{B}^{5}, \tilde{B}^{3}\right)$ and $\left(B^{5}, B^{3}\right)$ are diffeomorphic.

REMARK. It follows that each fibered 2-knot constructed in [As-Yo] is, in fact, the double of some invertible fibred ribbon disc.

4. Questions. If $K \subset S^{4}$ is a ribbon 2-knot of arbitrarily many fusions such that the commutator subgroup $G^{\prime}$ is finitely generated, is $K$ a fibred knot? An affirmative answer together with Theorem 3.0(A) of [Co] would settle, in many cases, a difficult unanswered question of Rapaport. (See the first two paragraphs of $\S 3$ in [Ra2].) This is so because many (weight 1) knot-like groups can be realised as fundamental groups of ribbon 2 -knots.

\section{REFERENCES}

[Ai] I. R. Aitchison, Isotoping and twisting knots and ribbons, Ph.D. Dissertation, Univ. of California at Berkeley, 1984.

[Ai-Ru] I. R. Aitchison and J. H. Rubinstein, Fibred knots and involutions on homotopy spheres, Four-Manifold Theory (Durham, N.H., 1982), Contemp. Math., vol. 35, Amer. Math. Soc., Providence, R. I., 1984.

[Ai-Si] I. R. Aitchison and D. S. Silver, On certain fibred ribbon disc pairs, Trans. Amer. Math. Soc. 306 (1988), 529551.

[Ak-Ki] S. Akbulut and R. C. Kirby, An exotic involution of $S^{4}$, Topology 18 (1979), 75-81.

[An-Cu] J. J. Andrews and M. L. Curtis, Free groups and handlebodies, Proc. Amer. Math. Soc. 16 (1985), $192-195$.

[As-Yo] K. Asano and K. Yoshikawa, On polynomial invariants of fibred 2-knots, Pacific J. Math. 97 (1981), 267269.

[Bl] R. C. Blanchfield, Intersection theory of manifolds with operators with applications to knot theory, Ann. of Math. (2) 65 (1957), 340 356.

[Bu] G. Burde, Alexanderpolynome Neuwirtscher Knoten, Topology 5 (1966), 321-330.

[Ca-Go] A. Casson and C. Mc. A. Gordon, A loop theorem for duality spaces and fibred ribbon knots, Invent. Math. 74 (1983), 119-137.

[Co] T. Cochran, Ribbon knots in $S^{4}$, J. London Math. Soc. (2) 28 (1983), 563-576.

[Fo-Mi] R. H. Fox and J. Milnor, Singularities of 2-spheres in 4-space and cobordism of knots, Osaka J. Math. 3 (1966), 257-267.

[Gi] P. Gilmer, Ribbon concordance and a partial order on S-equivalence classes, preprint 1983.

[Go1] C. Mc. A. Gordon, Some aspects of classical knot theory (J. C. Hausmann, ed.), Knot Theory, Lecture Notes in Math., vol. 685, Springer-Verlag, Berlin and New York, 1978, pp. 1-60.

[Go2] _ Ribbon concordance of knots in the 3-sphere, Math. Ann. 257 (1981), 157-170.

[Hi] R. L. Hitt, Handlebody presentations of knot cobordisms, Ph.D. Dissertation, Florida State Univ., 1977. 
[Ka] T. Kanenobu, Module d'Alexander des noeuds fibres et polynôme de Hosokawa des lacements fibres, Math. Sem. Notes Kobe University 9 (1981), 75-84.

[Ke-We] M. Kervaire and C. Weber, A survey of multidimensional knots Knot Theory (Proc., Plans-sur-Bex, 1977), Lecture Notes in Math., vol. 685, Springer-Verlag, Berlin and New York, 1978, pp. 61-134.

[Le] J. Levine, Some reults on higher dimensional knot groups, Knot Theory (Proc., Plans-sur-Bex, 1977), Lecture Notes in Math., vol. 685, Springer-Verlag, Berlin and New York, 1978, pp. 243-273.

[Ma] Y. Marumoto, On ribbon 2-knots of 1-fusion, Kobe Univ. Math. Sem. Notes 5 (1977), 59-68.

[Mi] J. Milnor, Infinite cyclic coverings, Conference on the Topology of Manifolds, Prindle, Weber and Schmidt, Boston, Mass., 1968, pp. 115-133.

[MKS] W. Magnus, A. Karrass, and D. Solitar, Combinatorial group theory, Dover, New York, 1976.

[Mo] J. M. Montesinos, Heegard diagrams for closed 4-manifolds, Geometric Topology (J. C. Cantrell, ed.), Academic Press, New York, 1979.

[Qu] C. V. Quach, Polynôme d'Alexander des noeuds fibres, C. R. Acad. Sci. Paris 289 (1979), 375-377.

[Qu-We] C. V. Quach and C. Weber, Une famille infinie de noeuds fibres cobordants a zero et ayant méme polynome d'Alexander, Comment. Math. Helv. 54 (1979), 562-566.

[Ra1] E. S. Rapaport, Note on Nielsen transformations, Proc. Amer. Math. Soc. 10 (1959), 228235.

[Ra2] — Knot-like groups, Ann. of Math. Studies, no. 84, Princeton Univ. Press, Princeton, N. J., 1975, pp. $119-133$.

[St] J. S. Stallings, Constructions of fibered knots and links, Proc. Sympos. Pure Math., vol. 32, part 2, Amer. Math. Soc., Providence, R. I., 1978, pp. 55-60.

[Su] D. W. Sumners, Invertible knot cobordisms, Comment. Math. Helv. 46 (1971), 240-256.

[Ta] O. Taussky, On a theorem of Latimer and MacDuffee, Canad. J. Math. 1 (1949), 300-302.

[Te] H. Terasaka, On null-equivalent knots, Osaka J. Math. 11 (1959), 95-113.

[Ya] T. Yanagawa, On ribbon 2-knots. I, II, Osaka J. Math. 6 (1969), 447-464.

[Yo] K. Yoshikawa, On fibering a class of n-knots, Kobe Math. Sem. Notes 9 (1981), 241-245.

[Ze] E. C. Zeeman, Twisting spun knots, Trans. Amer. Math. Soc. 115 (1965), 471-495.

Department of Mathematics, University of Melbourne, Parkville, VictoRIA 3052, Australia

Department of Mathematics and Statistics, University of South Alabama, Mobile, Alabama 36688 